\title{
Ameliorative effect of recombinant human lactoferrin on the premature ovarian failure in rats after cyclophosphamide treatments
}

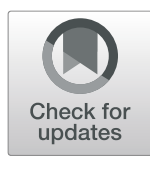

\author{
Shubin $\mathrm{Li}^{1,2+} \mathbb{D}$, Mengnan $\mathrm{Liu}^{1,3+} \mathbb{D}$, Hongmeng $\mathrm{Ma}^{1+}$, Qin $\mathrm{Jin}^{4} \mathbb{D}$, Yuzhen $\mathrm{Ma}^{2}$, Chunyu Wang ${ }^{1} \mathbb{D}$, Jingyu Ren ${ }^{1} \mathbb{D}$, \\ Gang Liu ${ }^{1,5^{*}}$ (D) and Yanfeng Dai ${ }^{*^{*}}$
}

\begin{abstract}
This study investigated the effect of recombinant human lactoferrin (rhLF) on the premature ovarian failure (POF) of rats. After cyclophosphamide treatments, the POF rats were divided into the following groups: normal control group (NC), low-dose group (LD), medium-dose group (MD) and high-dose group (HD) of rhLF. After drug administrations, the ovarian indexes and hormonal levels were detected. After follicle number count, the proliferation and apoptosis were analyzed with the expressions of genes related with oogenesis, reactive oxygen species (ROS) production and apoptosis detected, followed by the calculation of oxidative stress and protein expressions. After 4-hydroperoxy cyclophosphamide (4-HC) treatments, the effect of rhLF on the proliferation, ROS production and gene expressions of primary rat granulosa cells (GCS) cultured in vitro were detected. After mating, the fertilities of POF rats were recorded. The result showed that the rhLF administrations up-regulated the ovarian index with the number of developing follicles increased and the decreases of hormonal levels conferred. The Ki-67 intensities of the MD and HD groups were up-regulated with the Tunnel intensities decreased. The rhLF treatments significantly promoted the expression of oogenesis, antioxidant and anti-apoptosis related genes. The expression of Bax and Caspase 3 were decreased with the expression of BCl-2 up-regulated after rhLF administrations. The in vitro treatments of rhLF effectively conferred the toxicity of 4-HC on primary rat GCs. The fertility assessment showed the rhLF treatments up-regulated the offspring's' folliculogenesis, which confirmed the ameliorative role of rhLF on the POF damages via the inhibition of ROS production in GCs.
\end{abstract}

Keywords: rhLF, POF, Folliculogenesis, ROS production, Ovarian apoptosis

\section{Introduction}

During the past decades, the incidence and mortality of female reproductive tumors, including cervical carcinoma, endometrial carcinoma and ovarian carcinoma have been rapidly growing worldwide [3]. The complicated causes and recurrences of these malignant tumors

\footnotetext{
*Correspondence: 21408010@mail.imu.edu.cn; daiyf@imu.edu.cn

†Shubin Li, Mengnan Liu and Hongmeng Ma contributed equally to this work.

'College of Life Science, Inner Mongolia University, 235 West University Road, Hohhot 010021, Inner Mongolia, China

Full list of author information is available at the end of the article
}

have been formidable threats to women's well-beings. Although more efficient methodologies for tumor screening, diagnosis, surgery and treatments have been developed in recent years, the clinical outcomes and prognosis of female reproductive tumor patients remain to be further improved [35].

Nowadays, these current therapies for the surgery and radiotherapy have been the primary opinions for the clinical treatment of local and non-metastatic tumors, also, the anti-cancer therapies including chemotherapy, targeted therapies, hormone and biological therapies are the promising choices for the treatment of metastatic

(c) The Author(s). 2021 Open Access This article is licensed under a Creative Commons Attribution 4.0 International License, which permits use, sharing, adaptation, distribution and reproduction in any medium or format, as long as you give appropriate credit to the original author(s) and the source, provide a link to the Creative Commons licence, and indicate if changes were made. The images or other third party material in this article are included in the article's Creative Commons licence, unless indicated otherwise in a credit line to the material. If material is not included in the article's Creative Commons licence and your intended use is not permitted by statutory regulation or exceeds the permitted use, you will need to obtain permission directly from the copyright holder. To view a copy of this licence, visit http://creativecommons.org/licenses/by/4.0/ The Creative Commons Public Domain Dedication waiver (http://creativecommons.org/publicdomain/zero/1.0/) applies to the data made available in this article, unless otherwise stated in a credit line to the data. 
tumors [52]. However, due to the cellular toxicity, multidrug resistance (MDR) and reproductive disorders during the long term medication of anti-cancer drugs, the present application of anti-cancer drugs and recovery after drug administration still need to be further optimized for the development of more-effective personalized therapies $[9,10,54]$.

Among these numerous chemotherapy drugs targeting mitochondria activities [including apoptotic related proteins, cytochrome c, caspase 3, caspase 9 and reactive oxygen species (ROS)] [1, 69, 77], endoplasmic reticulum stress (including inositol-requiring enzyme $1 \alpha$, PKR-like ER kinase and activating transcription factor $6 \alpha$ ) [8], nucleus (including nucleolar phosphorprotein, nuclear pore complex and nuclear localization signal) [28, 64], tumor microenvironments $[26,41,71]$ and plasma membrane phospholipids [20,31, 32], cyclophosphamide (CTX), as an orally active alkylating agent, has been widely used as an utilized antineoplastic drug for the clinical treatment of ovarian, breast, testicular and hematological tumors [16].

However, the clinical applications of CTX have been reported with the oxidative stress-induced toxicities in vivo $[29,45,51,56,57,62]$, which further resulted in the irreversible damages of germ cells, cellular apoptosis, infertility and premature ovarian failure (POF). Different protective agents isolated from herbal and antioxidants reducing oxidative stress have been developed to prevent the reproductive toxicity of CTX [19]. Furthermore, lactoferrin (LF) has been reported with the suppression abilities of oxidative stress-induced toxicities and cellular apoptosis [17, 38, 42, 49, 50].

As a natural pleiotrophic glycoprotein from the transferrin family, mammalian LF is mainly produced by epithelial cells, neutrophil precursors and placenta [7]. The presence of mammalian LF in these biological fluids including tears, saliva, pancreatic fluid, nasal, semen, urine and mostly abundant in mammalian milk further regulated the following biological processes as iron transport, anti-microbial defense and immune system regulation $[34,60,67,74]$. And the large scale manufacture of bovine LF from skim milk and whey has been established from the 1990s [7]. In 2006, bovine LF has been applied as supplements to cosmetics, beverages, cosmetics, infant formula, pet foods and yogurt in Japan [66], meanwhile, the commercial infant formulas enriched with bovine LF has been available in Indonesia, South Korea and Spain [7].

In addition, due to the protective properties of antioxidant and anti-cancer, recombinant human lactoferrin (rhLF) modulates the production of cytokines with regards to cancer progression $[21,27,68]$.

However, the therapeutic effect of rhLF on the mammalian reproductive abilities after CTX treatments via the suppression of oxidative stress-induced toxicities and cellular apoptosis remains unclear. Therefore, the aim of this study was to investigate the potential effects of oral rhLF administration on the therapeutic effect of rat POF-damages caused by CTX treatments. After the treatment of 4-hydroperoxy cyclophosphamide (4-HC, as the active in vitro metabolite of CTX), the effect of rhLF treatments on the proliferation, ROS production and gene expression levels of primary rat granulosa cells (GCs) cultured in vitro were detected to further confirm the ameliorative effect of rhLF on the POF damages and provide further basis for the clinical applications of rhLF.

\section{Results}

\section{The physiological states of CTX-induced POF rats after rhLF administrations}

First of all, the body weight of each rat after CTX treatments was examined to ensure the availabilities of the following experimental data and the results showed there were no significant differences among the body weight of 80 rats after CTX treatments.

As shown in Fig. 1a, the body weight of rats in all rhLF administration groups were remarkably higher in comparison with the NC group $(275.38 \pm 9.61)(P<0.05)$, meanwhile, there was no significant difference between the body weight of the MD $(315.07 \pm 4.44 \mathrm{~g})$ and $\mathrm{HD}$ groups $(322.63 \pm 11.96 \mathrm{~g})$.

As shown in Fig. 1b, the abnormalities of estrous cycles in rats caused by CTX treatments were significantly reverted by rhLF administrations in a dose-dependent manner $(P<0.05)$. Furthermore, the results of ovarian index analyses showed that the ovarian indexes in all rhLF administration groups were significantly increased (Fig. 1c) in comparison with the NC group $(P<0.05)$, which indicated that the rhLF administrations significantly ameliorated the abnormal estrous cycles and improved the ovarian development of CTX-induced POF rats.

Furthermore, the hormonal levels of estradiol, progesterone and $\mathrm{AMH}$ in rats from different groups were analyzed to investigate the effect of rhLF administrations on the secretion of reproductive hormones. As shown in Fig. 1d, the results of estradiol level analyses showed that the estradiol levels of rats were significantly increased from $341.26 \pm 12.73 \mathrm{pg} / \mathrm{mL}$ for the NC group, $395.80 \pm$ $13.66 \mathrm{pg} / \mathrm{mL}$ for the LD group, $479.01 \pm 13.26 \mathrm{pg} / \mathrm{mL}$ for the MD group to $522.28 \pm 20.09 \mathrm{pg} / \mathrm{mL}$ for the HD group $(P<0.05)$.

In addition, the progesterone levels of rats in the rhLF administration groups were significantly higher than the NC group $(0.74 \pm 0.08 \mathrm{ng} / \mathrm{mL})$, meanwhile, the progesterone levels of rats in the rhLF administration groups were positively correlated with the concentration of rhLF administration $(P<0.05)$ (Fig. 1e). 

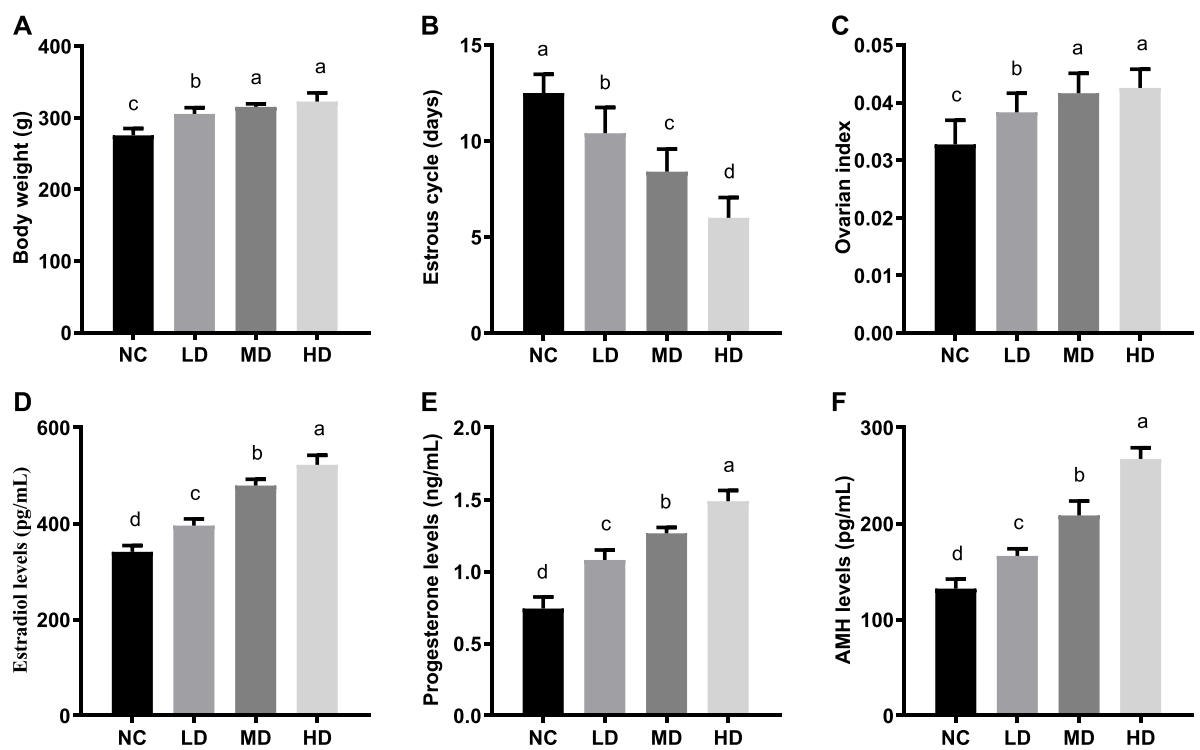

Fig. 1 The physiological states of CTX-induced POF rats after rhLF administrations. a The body weight of CTX-induced POF rats in different groups after rhLF administrations; $\mathbf{b}$ The estrous cycle of CTX-induced POF rats in different groups after rhLF administrations; c The ovarian index of CTX-induced POF rats in different groups after rhLF administrations. $\mathbf{d}$ The estradiol levels of CTX-induced POF rats in different groups after rhLF administrations; e The progesterone levels of CTX-induced POF rats in different groups after rhLF administrations; $\mathbf{f}$ The AMH levels of CTXinduced POF rats in different groups after rhLF administrations. Note: In each panel, labeling with the different letter in each column indicates significant differences between different groups $(P<0.05)$

Furthermore, the AMH levels of rats in the rhLF administration groups significantly increased from $132.26 \pm 9.81$ $\mathrm{pg} / \mathrm{mL}$ for the NC group, $166.31 \pm 7.35 \mathrm{pg} / \mathrm{mL}$ for the LD group, $208.44 \pm 14.93 \mathrm{pg} / \mathrm{mL}$ for the $\mathrm{MD}$ group to $267.32 \pm 11.68 \mathrm{pg} / \mathrm{mL}$ for the HD group $(P<0.05)$.

These above results further confirmed that the rhLF administrations improved the abnormal secretion of reproductive hormones in a dose dependent manner, suggesting that the rhLF administrations could significantly ameliorated the physiological disorders of CTX-induced POF rats.

\section{The histological analyses of ovaries from CTX-induced POF rats after rhLF administrations}

To further analysis the effect of rhLF administrations on the physiological state of ovaries from CTX-induced POF rats, the histological analyses of HE were conducted, followed by follicle number count.

As shown in Fig. 2a, an abnormal histology with ovarian interstitial fibrosis, inflammatory cell infiltration and vessel dilation was observed in the rat ovaries of the NC group. The histological results showed that after rhLF administrations, the ovarian damages caused by CTX treatments were significantly alleviated in the rat ovaries.

And the results of follicle number count showed that the number of developing follicles at different stages (primary, secondary and antral follicles) in the ovaries of rhLF administration groups was significantly increased in comparison with the NC group $(P<0.05)$, however, the number of atretic follicles in the ovaries of the NC group was significantly higher than all rhLF administration groups $(P<0.05$, Fig. 2b). Meanwhile, there were no significant differences between the number of developing follicles (regardless of primary, secondary or antral follicles) in the MD and HD groups. In addition, the number of corpus lutea in all rhLF treatment groups was significlantly up-regulated in comparison with the NC group $(P<0.05$, Fig. $2 \mathrm{~b})$.

\section{The proliferative abilities of follicles from CTX-induced POF rats after rhLF administrations}

As shown in Fig. 3, the Immunohistochemistry (IHC) staining results of $\mathrm{Ki}-67$ protein showed that the positive intensities of Ki-67 (mainly detected in GCs) in all rhLF administration groups were significantly higher compared with that of the NC group $(P<0.05)$, in addition, there was no significant difference between the Ki-67 positive intensities of the MD group and HD groups, which further confirmed that the rhLF administrations significantly promoted the proliferations of ovarian cells after CTX treatments.

\section{The apoptosis levels of follicles from CTX-induced POF rats after rhLF administrations}

Tunnel staining was further conducted to investigate the effect of rhLF administrations on the follicular apoptosis levels. As presented in Fig. 4, the majority of apoptosis cells mainly located in GCs, furthermore, the Tunnel rates as cellular apoptosis rates (\%) in the rhLF 

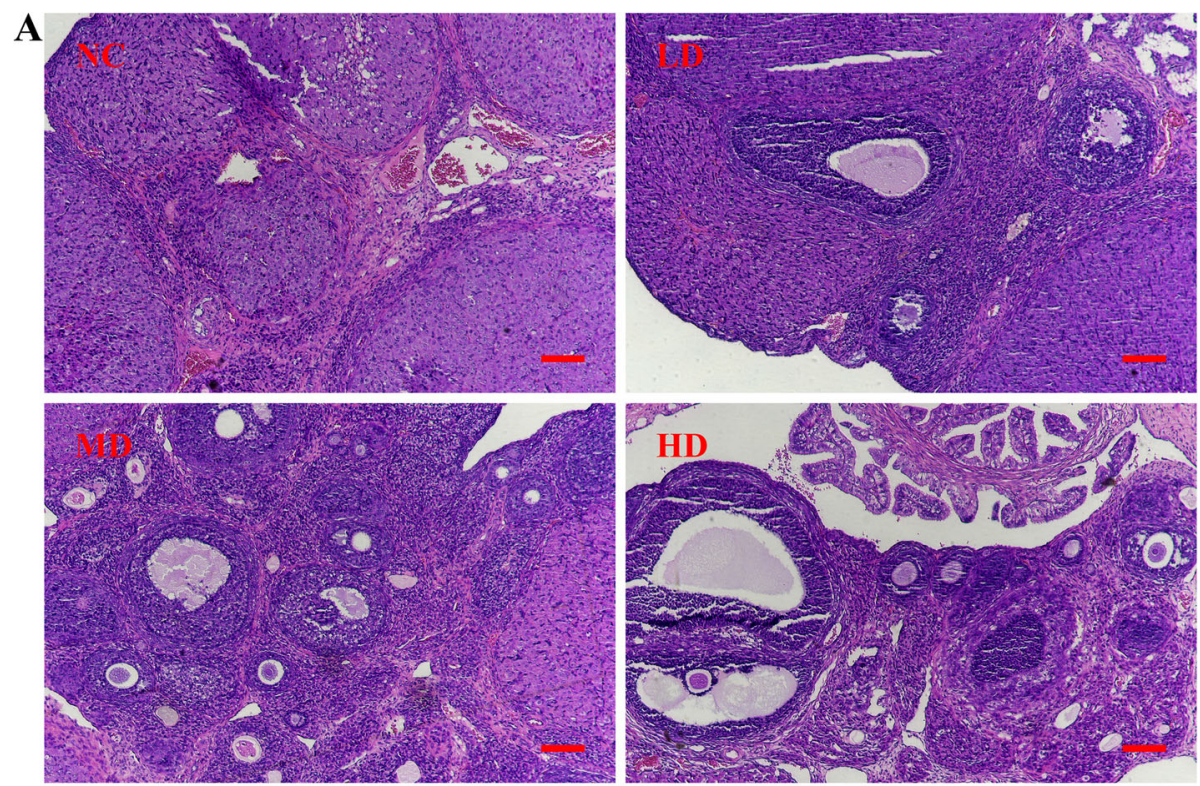

B

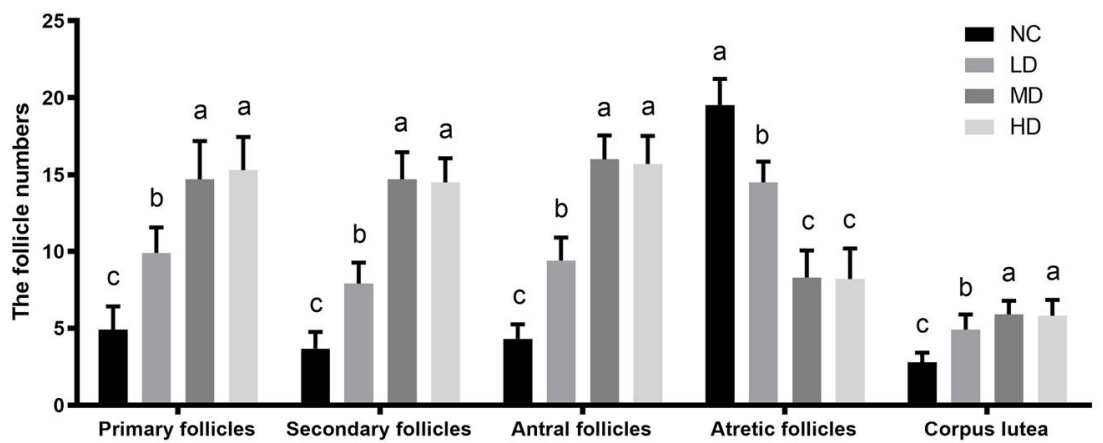

Fig. 2 The histological analyses of ovaries from CTX-induced POF rats after rhLF administrations. a The representative HE staining results of ovaries from CTX-induced POF rats in different groups after rhLF administrations; Scale bar $=100 \mu \mathrm{m}$; $\mathbf{b}$ The follicle number of ovaries from CTXinduced POF rats in different groups after rhLF administrations. Note: In each panel, labeling with the different letter in each column indicates significant differences between different groups $(P<0.05)$

administration groups were significantly reduced in comparison with the NC group $(P<0.05)$.

The antioxidant abilities of ovaries from CTX-induced POF rats after rhLF administrations

As shown in Fig. 5a, the results of ovarian ROS production levels showed that the ovarian ROS production levels were significantly reduced from $247.01 \pm 8.30 \mathrm{IU} /$ $\mathrm{mL}$ for the NC group, $207.69 \pm 6.48 \mathrm{IU} / \mathrm{mL}$ for the $\mathrm{LD}$ group, $182.52 \pm 7.01 \mathrm{U} / \mathrm{mL}$ for the $\mathrm{MD}$ group to $166.90 \pm 4.29 \mathrm{U} / \mathrm{mL}$ for the $\mathrm{HD}$ group in a dose dependent manner $(P<0.05)$. Furthermore, the rhLF administration significantly promoted the ovarian SOD levels from $66.97 \pm 4.77 \mathrm{IU} / \mathrm{g}$ for the NC group, $87.56 \pm$ $3.18 \mathrm{IU} / \mathrm{g}$ for the MD group, $94.74 \pm 2.93 \mathrm{IU} / \mathrm{g}$ for the LD group to $94.98 \pm 2.80 \mathrm{IU} / \mathrm{g}$ for the HD group $(P<$ 0.05). The ovarian CAT levels in all rhLF administration groups (as $7.32 \pm 0.35 \mathrm{IU} / \mathrm{g}$ for the LD group, $8.91 \pm 0.45$
$\mathrm{IU} / \mathrm{g}$ for the MD group and $11.25 \pm 0.67 \mathrm{IU} / \mathrm{g}$ for the HD group) were significantly higher in comparison with the $\mathrm{NC}$ group $(4.61 \pm 0.28 \mathrm{IU} / \mathrm{g}) \quad(P<0.05)$. The ovarian MDA level of the NC group $(1.42 \pm 0.08 \mu \mathrm{M} / \mathrm{g})$ was significantly higher than the LD $(1.14 \pm 0.09 \mu \mathrm{M} / \mathrm{g}), \mathrm{MD}$ $(0.75 \pm 0.08 \mu \mathrm{M} / \mathrm{g})$ and $\mathrm{HD}$ groups $(0.64 \pm 0.09 \mu \mathrm{M} / \mathrm{g})$, respectively $(P<0.05)$.

The results of RT-PCR showed that the transcription levels of these specific genes for oogenesis as Oct4, Bmp15 and Gdf9 were significantly increased in all rhLF administration groups in a dose dependent manner $(P<$ 0.05 , Fig. $5 b$ ), which was similar with the expression patterns of these specific genes of GCs as Inhibin alpha, Foxo3 and Fshr in all rhLF administration groups. Furthermore, the expression levels of these specific genes for the antioxidant abilities as Sod2, Catalase and Gpx1 were significantly increased after rhLF administrations, which further confirmed the antioxidant characteristic of 


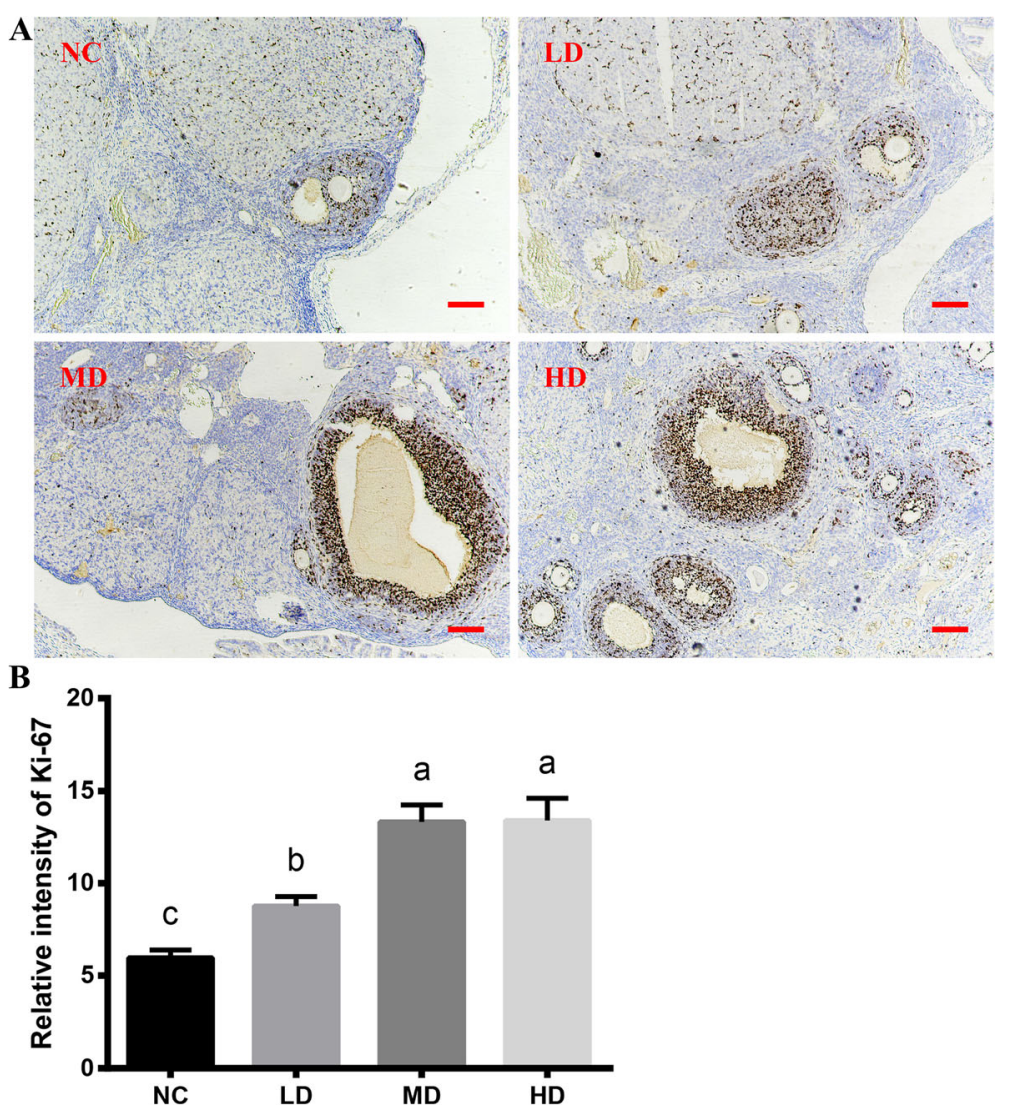

Fig. 3 The Immunohistochemical staining results of ovaries from CTX-induced POF rats after rhLF administrations. a The representative Immunohistochemical staining of Ki-67 of ovaries from CTX-induced POF rats in different groups after rhLF administrations. Scale bar $=100 \mu \mathrm{m}$. $\mathbf{b}$ The relative expression levels of Ki-67 of ovaries from CTX-induced POF rats in different groups after rhLF administrations. Note: In each panel, labeling with the different letter in each column indicates significant differences between different groups $(P<0.05)$

rhLF on POF ovaries after CTX treatments $(P<0.05)$. After rhLF administrations, the ovarian expression levels of $B a x$ was significantly decreased in comparison with the NC group $(P<0.05)$. Meanwhile, the ovarian expression levels of $\mathrm{Bcl}-2$ in all rhLF administration groups were significantly higher than the $\mathrm{NC}$ group $(P<0.05)$, however, there was no significant difference in the expression level of $\mathrm{Bcl}-2$ between the MD and HD groups.

To further confirm the effect of rhLF administrations on the ovarian apoptosis process, the ovarian expression levels of $\mathrm{Bcl}-2$, Bax and Caspase 3 were detected by Western blot. As shown in Fig. 5c, significantly decreased levels of Bax and Caspase 3 were found in all rhLF administration groups compared with the NC group $(P<0.05)$, meanwhile, the expression levels of Bcl2 in all rhLF administration groups were significantly higher than the NC group $(P<0.05)$, which further confirmed that the rhLF administrations significantly inhibited the ovarian apoptosis process.

These above results further indicated that the rhLF administration significantly inhibited the ovarian oxidative stress levels and enhanced the ovarian antioxidant abilities after CTX treatments.

\section{The ROS production, proliferation and gene expression} levels of primary rat GCs after 4-HC and rhLF treatments To confirm the effect of rhLF treatments on the ROS production levels of primary rat GCs, ROS staining of primary rat GCs were analyzed with the representative images of DCFH-DA staining shown in Fig. 6a. As shown in Fig. 6a, the fluorescence intensities of DCFHDA in primary rat GCs were significantly reduced in all rhLF groups compared with the rhLF 0 group $(P<0.05)$.

Furthermore, the fluorescence intensities of Edu staining in primary rat GCs were significantly enhanced in the rhLF 200 and rhLF 300 groups, compared with the rhLF 0 and rhLF 100 groupS $(P<0.05)($ Fig. 7$)$, indicating that the treatment of rhLF significantly inhibited the ROS production levels and promoted the proliferative potentials of primary rat GCs during the in vitro treatment of 4-HC.

In addition, the RT-PCR results of primary rat GCs during the in vitro treatments of 4-HC and rhLF confirmed that the rhLF treatments significantly up- 

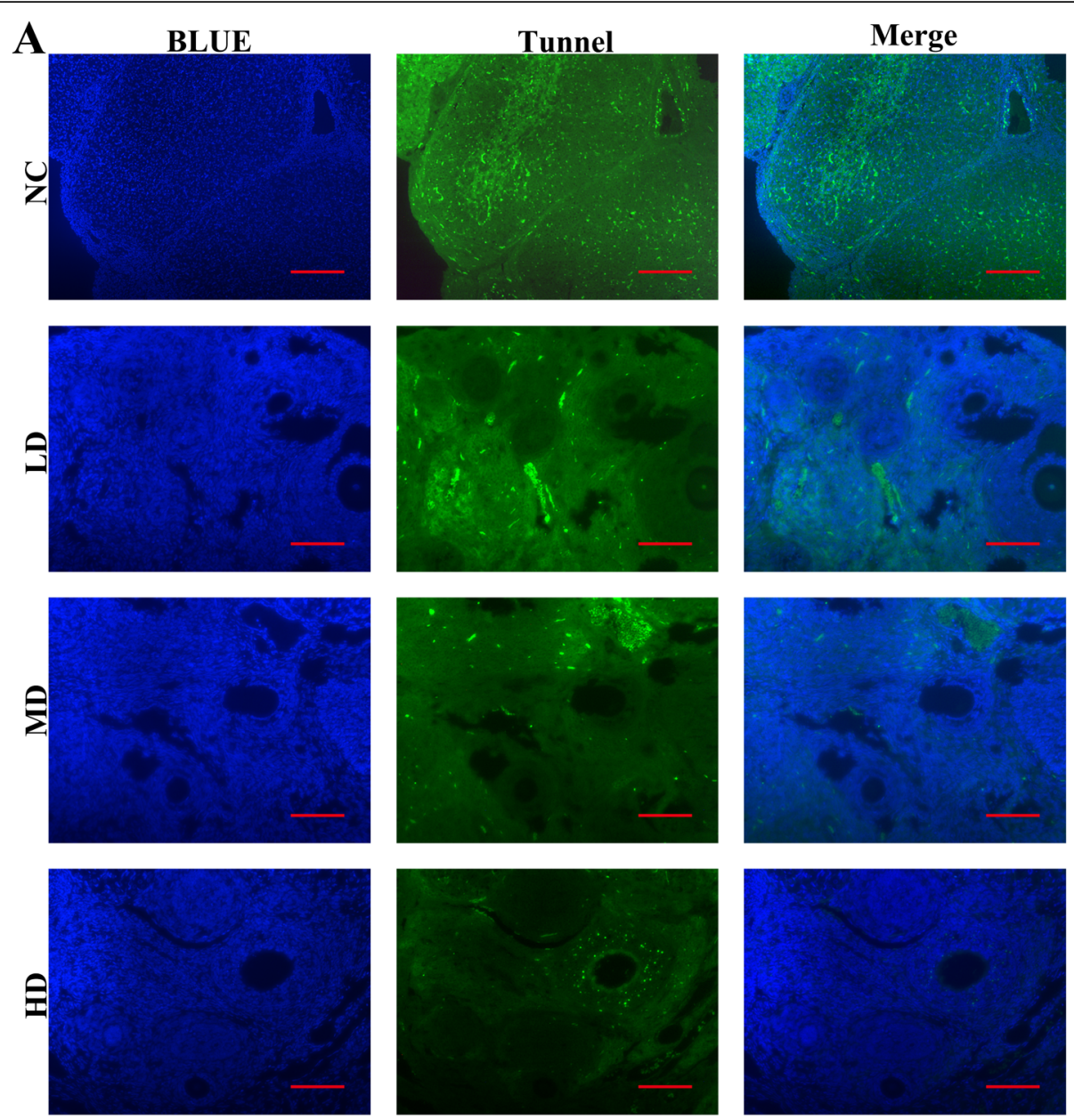

\section{B}

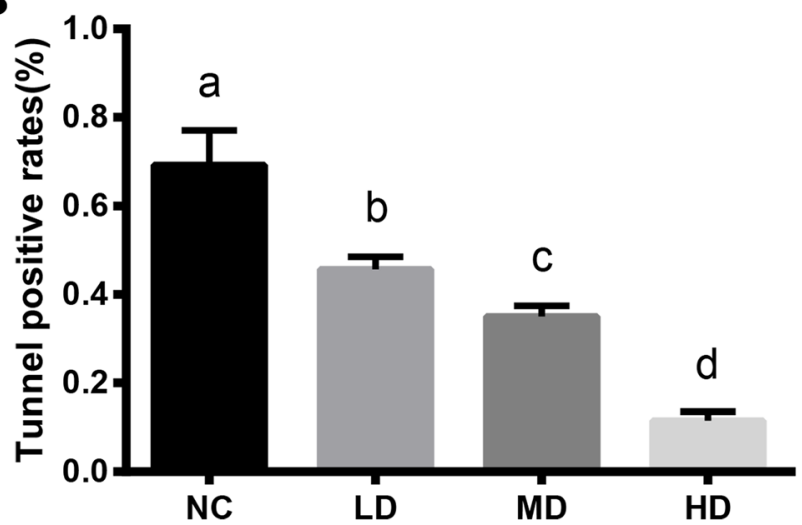

Fig. 4 The Tunnel staining results of ovaries from CTX-induced POF rats after rhLF administrations. a The representative Tunnel (grenn) and DAPI (blue) staining results of follicles from CTX-induced POF rats in different groups after rhLF administrations; Scale bar $=200 \mu \mathrm{m}$; $\mathbf{b}$ The relative Tunnel rates (\%) of follicles from CTX-induced POF rats in different groups after rhLF administrations. Note: Labeling with the different letter in each column indicates significant differences between different groups $(P<0.05)$.

regulated the cellular expression levels of FOXL2, Inhibin alpha, SOD2, Catalase, GPx and Bcl-2 in a dose dependent manner (Fig. 8, $P<0.05$ ). On the other hand, the expression levels of $B A X$ in the $\mathrm{NC}$ group were significantly higher than that of all rhLF groups $(P<0.05)$.
The fertility abilities assessment of CTX-induced POF rats after rhLF administrations

To further confirm the effect of rhLF administrations on the fertility abilities of CTX-induced POF rats, the assessments of fertility abilities were conducted. As 

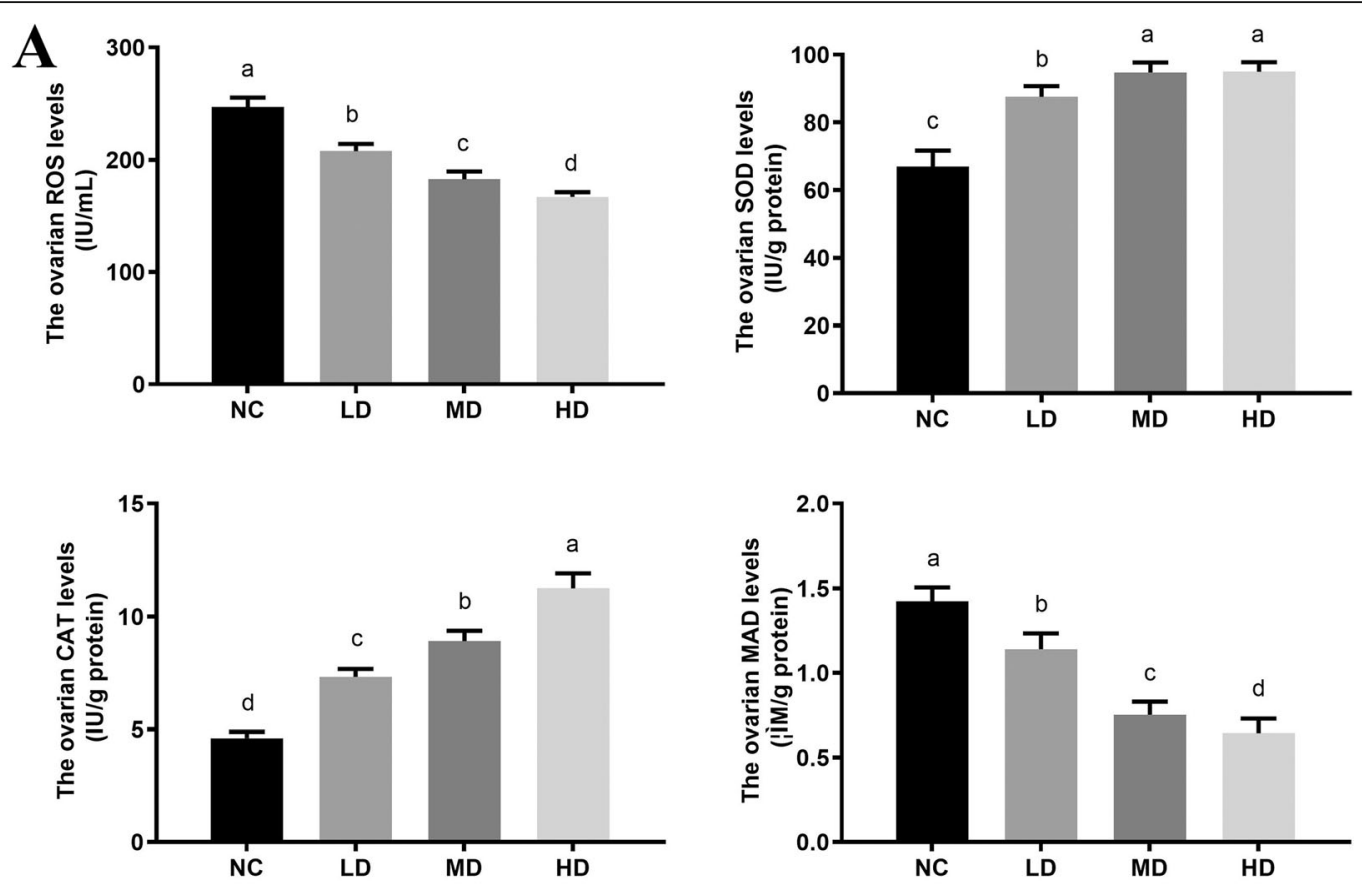

B
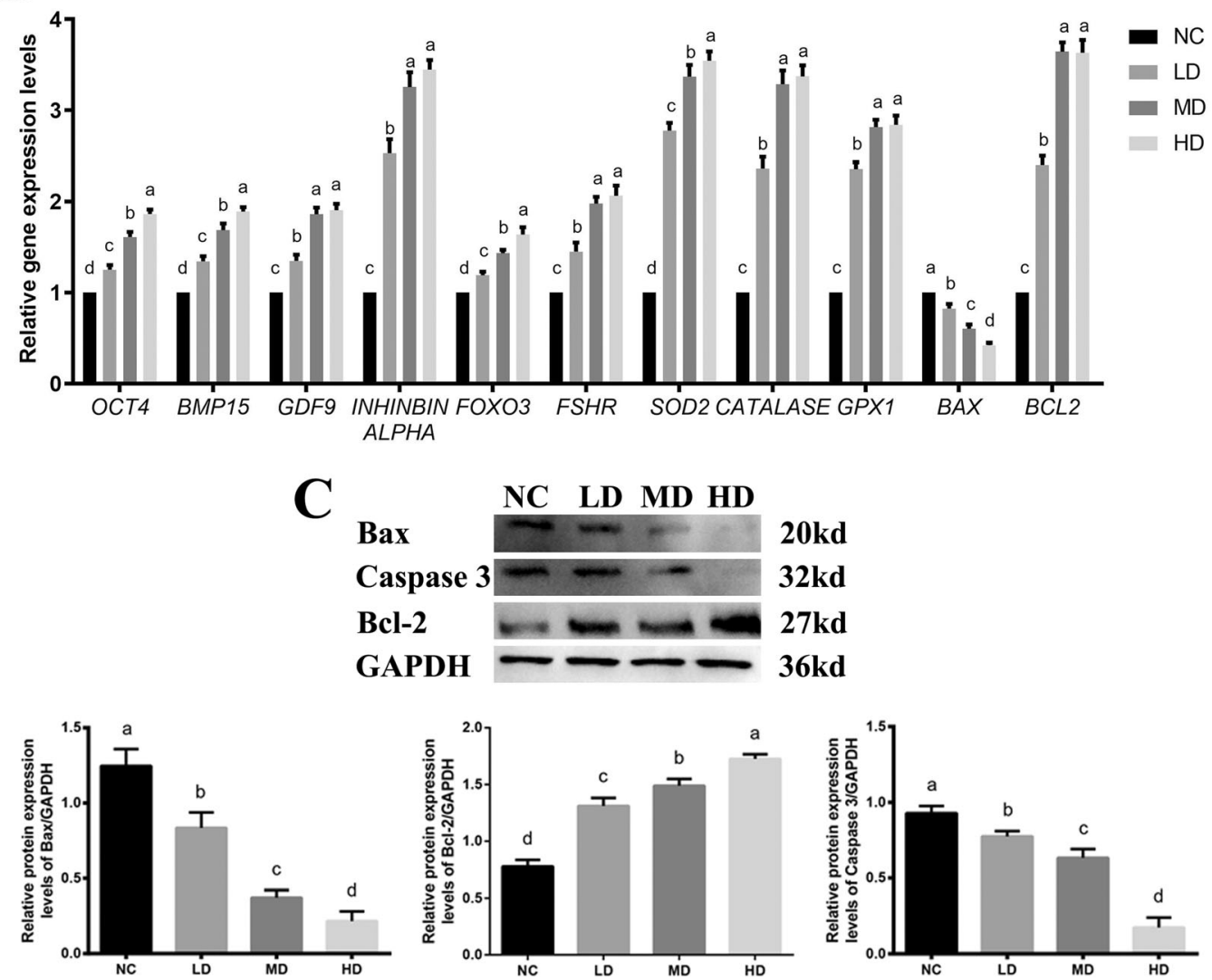

Fig. 5 The antioxidant abilities of CTX-induced POF rats after rhLF administrations. a The ovarian ROS production levels (IU/mL), SOD levels (IU/g), TAOC levels (IU/g) and MDA levels $(\mu \mathrm{M} / \mathrm{g})$ in different groups after rhLF administrations. b The relative gene expression levels in ovaries from CTX-induced POF rats after rhLF administrations. c The protein expression patterns in ovaries from CTX-induced POF rats after rhLF administrations. Note: In each panel, labeling with the different letter in each column indicates significant differences between different groups $(P<0.05)$. 


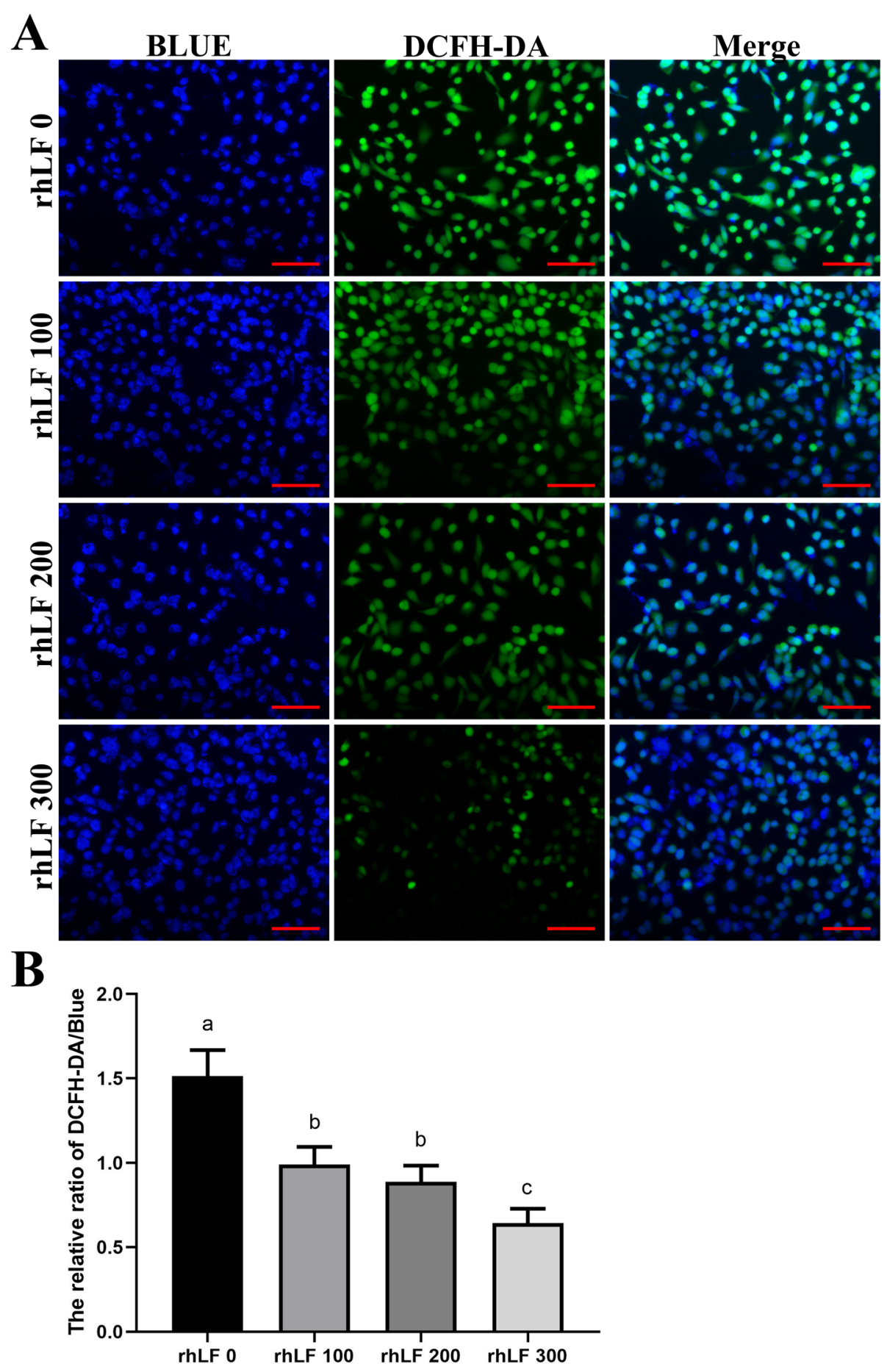

Fig. 6 The ROS staining results of primary rat GCs after 4-HC and rhLF treatments. a The representative ROS staining results of primary rat GCs in different groups after rhLF treatments. Scale bar $=100 \mu \mathrm{m}$. $\mathbf{b}$ The relative densities of ROS staining of primary rat GCs in different groups after rhLF treatments. Note: In each panel, labeling with the different letter in each column indicates significant differences between different groups $(P<0.05)$

shown in Table 1, the results showed that the rhLF administration resulted in a significant increase in the litter sizes in comparison with the NC group $(P<$ $0.05)$, meanwhile there was no significant difference in the litter sizes among all rhLF administration groups.

As shown in Fig. 9, the body weight and ovarian index of female offspring in all rhLF administration groups 


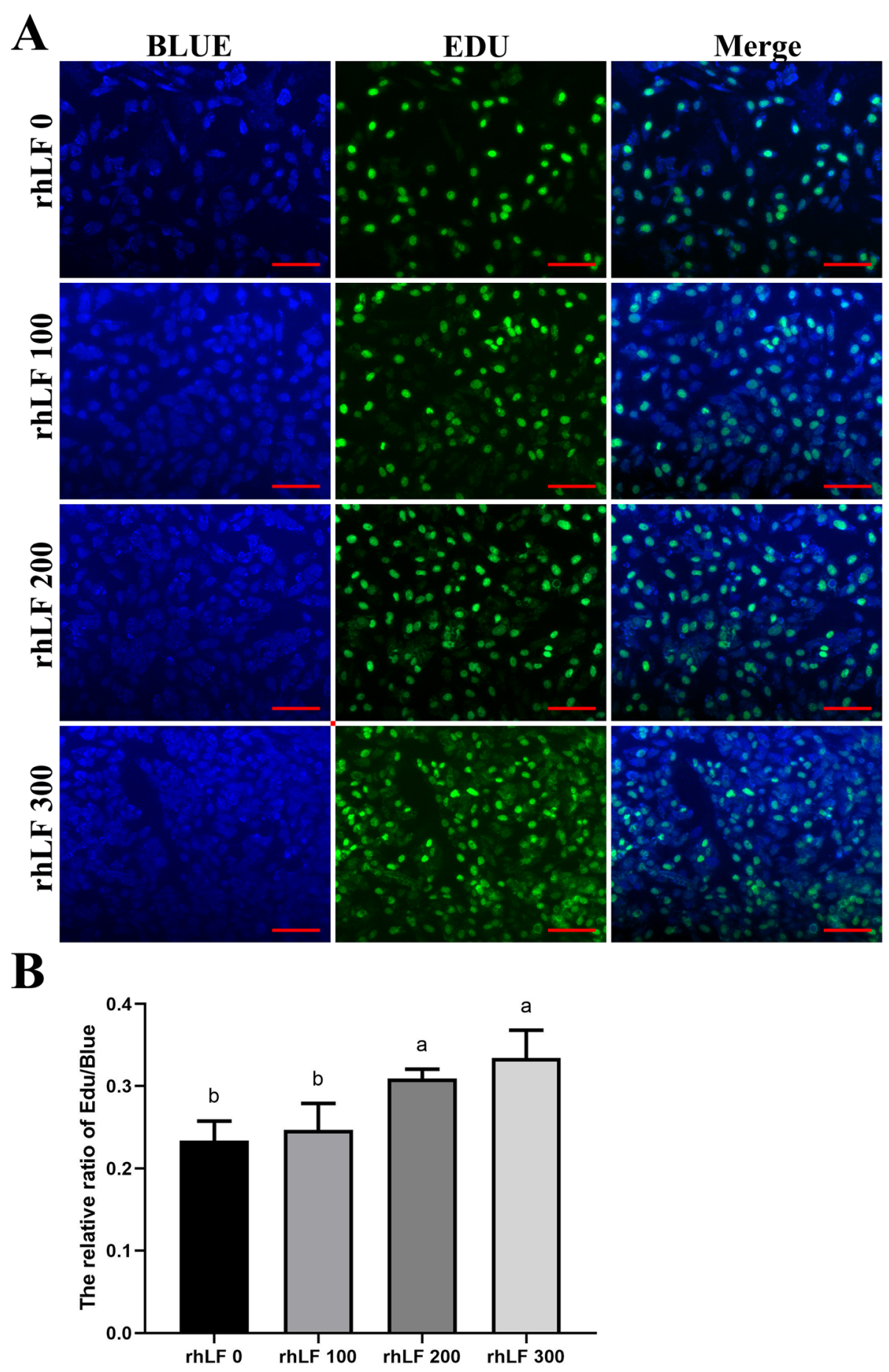

Fig. 7 The EDU staining results of primary rat GCs after 4-HC and rhLF treatments. a The representative EDU staining results of primary rat GCs in different groups after rhLF treatments. Scale bar $=100 \mu \mathrm{m}$. $\mathbf{b}$ The relative densities of EDU staining of primary rat GCs in different groups after rhLF treatments. Note: In each panel, labeling with the different letter in each column indicates significant differences between different groups $(P<0.05)$

were significantly higher than the NC group $(P<0.05)$. Furthermore, the hormonal levels of estradiol and progesterone in all rhLF administration groups were significantly higher that the $\mathrm{NC}$ group $(P<0.05)$.
The HE staining results (Fig. 10) further confirmed that the oogenesis process in the offspring ovaries of all rhLF administration groups were up-regulated than that the NC group, however, the exact number of 


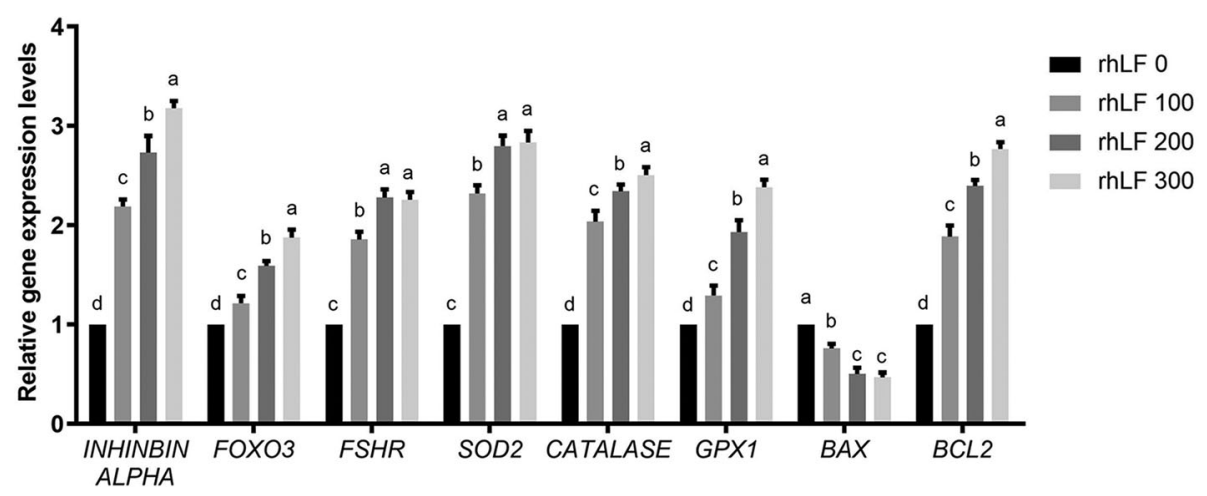

Fig. 8 The gene expression levels of primary rat GCs after 4-HC and rhLF treatments. Note: In each panel, labeling with the different letter in each column indicates significant differences between different groups $(P<0.05)$

developing follicles and atretic follicles remains to be calculated.

\section{Discussion}

Although the chemotherapy treatments effectively improved the prognosis of female cancer patients, the side effects and safety concerns of chemotherapy-induced POF including the risk of psychological distress, autoimmune disorders, infertility, ischemic cardiac diseases, sexual disorders, amenorrhea and osteoporosis still needs to be resolved $[2,79]$.

During the past decades, the clinical treatments for chemotherapy-induced POF patients have been developed rapidly with the treatments including hormone replacement therapy, stem cell therapy, ovary cryopreservation and donor-oocyte in vitro fertilization (IVF) technology already been applied for the POFrelated symptoms [81], however, the clinical treatments of chemotherapy-induced POF patients remains unsatisfactory due to the complex pathogenesis of POF [11] or the increased risks for breast and endometrial cancers [33].

In our study, the effect and antioxidant related mechanisms of rhLF on the CTX-induced POF rats were analyzed to investigate the potential role of rhLF on the clinical treatment of cancer patients after CTX treatments.

Nowadays, many antioxidant supplements including vitamins, minerals, and polyphenols have been applied to reverse the oxidative stress-related damages and diseases caused by CTX treatments [18, 78, 84]. However, the safety of antioxidant active substances for the ovarian damages caused by oxidative stress needs further investigations.

In 2004, recombinant mouse LF receptor was found to be expressed in the various mouse tissues including brain, small intestinal epithelium, stomach, kidney and ovary [58]. In 2007, the existence of LF in murine follicular fluid and the correction between its concentration and embryonic development rates has been reported [75]. In 2008, the effect of bovine LF on the CTXtreated mouse ovaries was evaluated, and the results showed that the bovine LF administration rescued the CTX-induced ovarian damages with the gene expression level of Adamts1 and Sohlh1 and the protein expression of PCNA remarkably up-regulated [24]. In 2008, Stevenson et al reported that after oral supplements of bovine LF for $7 \mathrm{~d}$, the total, helper and cytotoxic T-cell activation and hydrophilic antioxidant status of male volunteers were significantly promoted [44].

Furthermore, the antioxidant abilities of LF have been reported with the mitigation of liver damage, oxidative stress and hepatotoxicity caused by carbon tetrachloride treatments [17]. In addition, the hLF administration effectively suppressed the excessive iron accumulation caused by the 1-methyl-4-phenyl-1,2,3,6-tetrahydropyridine (MPTP) treatments and the up-regulation of divalent metal transporter (DMT1) and transferrin receptor

Table 1 The fertility index and litter sizes in different groups after rhLF administrations

\begin{tabular}{lllll}
\hline & NC & LD & MD & HD \\
\hline Mating index & $10 / 10(100 \%)$ & $10 / 10(100 \%)$ & $10 / 10(100 \%)$ & $10 / 10(100 \%)$ \\
Fertility index & $7 / 10(70 \%)$ & $10 / 10(100 \%)$ & $10 / 10(100 \%)$ & $10 / 10(100 \%)$ \\
Mean litter size & $5.67 \pm 1.80 \mathrm{~b}$ & $14.22 \pm 1.56 \mathrm{a}$ & $14.44 \pm 1.33 \mathrm{a}$ & $15.33 \pm 1.32 \mathrm{a}$ \\
\hline
\end{tabular}




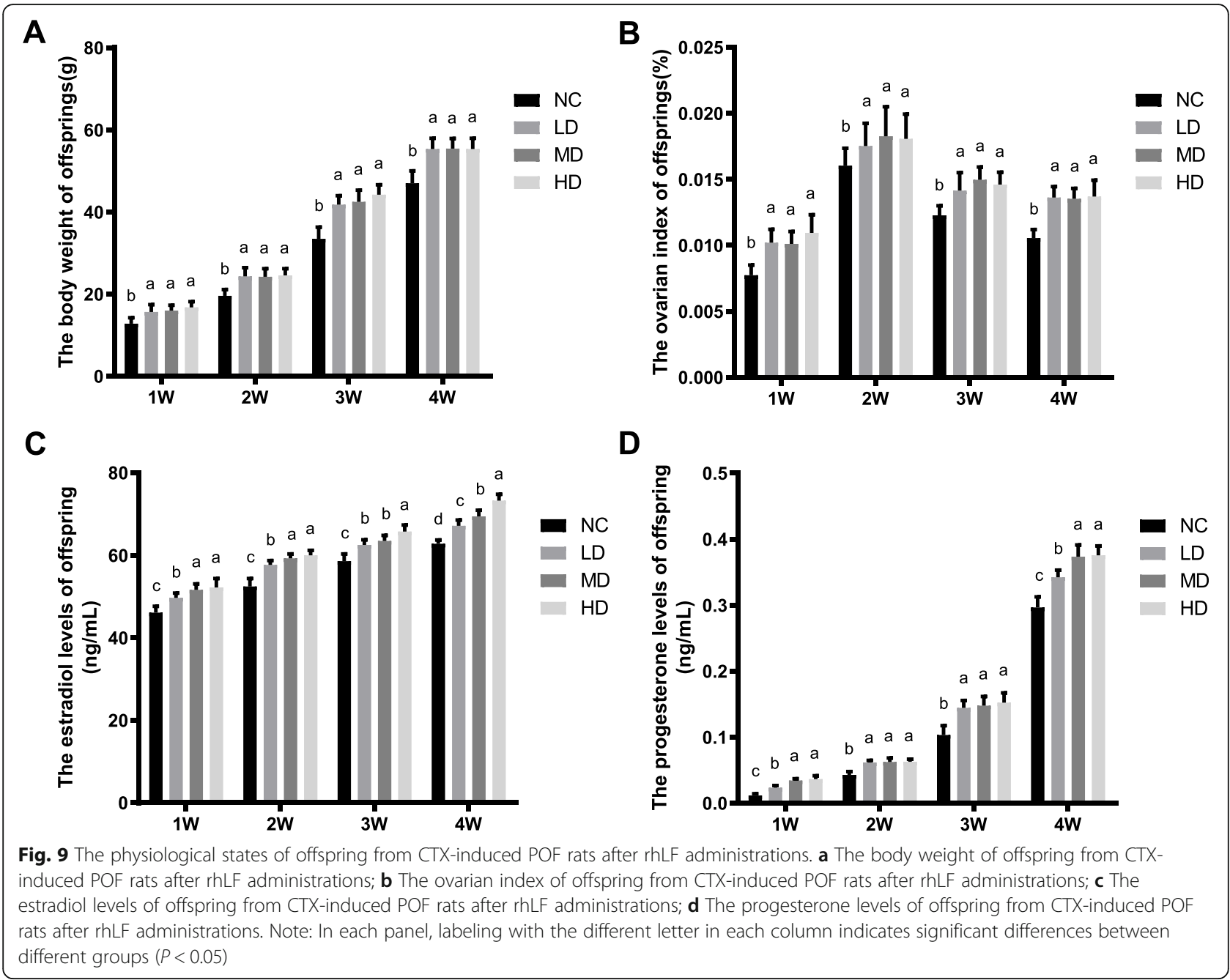

(TFR), which subsequently improved the activity of antioxidant enzymes including SOD1 and GPX4 and decreased the ROS production levels [72].

In 2018, Ateya et al found that the LF administration significantly improved the levels of serum catalase, nitric oxide and GSH with a significantly down-regulated MDA level in a model of ossimi lambs, in addition, the immune-modulatory gene levels of TNF- $\alpha, I L-1 \beta, I L-6$ and $I L-10$ were significantly improved after LF administrations [15]. However, the antioxidant effect of rhLF against CTX-induced POF in rat ovaries and the potential target needs further investigations.

In our study, the antioxidant effects of rhLF against CTX-induced POF rats were confirmed with improved histological parameters, reduced lipid peroxidation process, increased antioxidant activities and decreased cellular apoptosis levels. And the in vitro assessment of rhLF against 4-HC-induced cytotoxicity further confirmed the main target of rhLF against CTX-induced POF damages was GCs. Furthermore, the fertility abilities of CTX-induced POF rats after rhLF administrations were resumed.

During the process of CTX treatments in vivo, the elevated oxidative stress in ovarian cells is significantly related with the apoptosis process of mice and rat GCs [46]. The histopathological staining results (including HE, Immunohistochemistry and Tunnel staining) in this study further indicated that the ovarian damages caused by CTX treatments were mainly located in rat GCs, instead of oocytes. Furthermore, the rhLF administration effectively reversed the apoptosis levels of rat GCs, which was confirmed by the observations that the gene expression levels of Foxo3, Inhibin alpha and Fshr, the hormonal levels of estradiol, progesterone and $\mathrm{AMH}$ (mainly secreted by GCs) after rhLF administration were significantly up-regulated.

During the oogenesis process in vitro, GCs maintains the ovarian local microenvironments for the development and maturation of oocytes via the secretion of mediators including follicle stimulating hormone (FSH), 


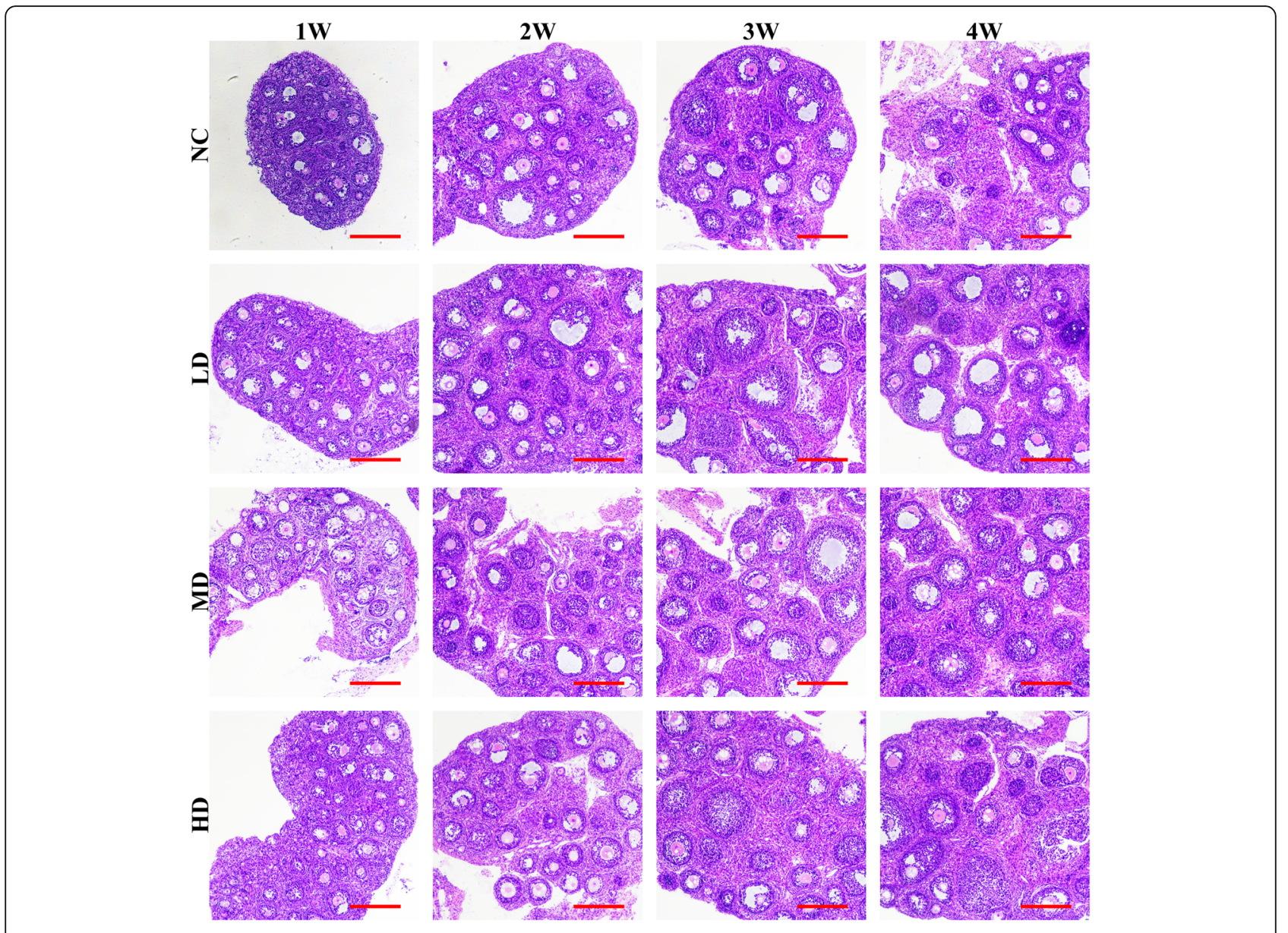

Fig. 10 The representative HE staining results of offspring's ovaries in different groups after rhLF administrations; Scale bar =200 $\mu \mathrm{m}$

luteinizing hormone $(\mathrm{LH})$, prostaglandins, chemokines and cytokines [12, 65]. The apoptosis of GCs has been treated as a leading contributor to POF in women $[4,30$, 39]. During the entire process of oogenesis from embryonic stem cells (ESCs) and induced pluripotent stem cells (iPSCs), the GCs originated from the gonadal somatic cells play important roles on the growth and function of oocytes [22, 23], which further indicated the important roles of GCs during mammalian oogenesis.

The primary rat GCs was further applied to confirm the ameliorative effect of rhLF on POF damages. And the results showed that the rhLF treatments significantly protected the primary rat GCs from the 4- $\mathrm{HC}$ treatments via the inhibition of ROS production and the promotion of cellular proliferation, which was consistent with the in vivo results of rhLF administrations. In addition, the high expression levels of Bax after 4-HC treatments revealed the increased levels of cellular apoptosis, which was consistent with these previous studies about the exposure to chemotherapy drugs in vitro and high levels of GCs apoptosis [5, 14, 55, 82], and our results further confirmed the main target of rhLF treatments during POF was GCs. However, due to the complex in vivo microenvironments including oocytes and follicular cells (GCs, theca cells and stroma cells) in mammalian ovaries, the ameliorative target of rhLF on the POF damages needs more detailed in vitro investigations.

Furthermore, the estrous cycles and fertility abilities of POF rats after rhLF administrations were analyzed for the first time and the results showed that rhLF significantly reduced the abnormalities of estrous cycle caused by CTX treatments. In addition, the litter sizes of the CTX-induced POF rats after rhLF administrations were significantly up-regulated. Due to these altered maternal endocrine-metabolic environments and pregnancyrelated complications of POF, the female fetus and longterm offspring health were adversely affected [13, 53], therefore, the effect of rhLF on the intergenerational transmission process of POF needs to be investigated to further confirm the safety of rhLF for clinical applications. 


\section{Conclusion}

This study provided direct evidences that the oral administrations of rhLF reduced the symptoms of CTX-induced POF by increasing the hormonal levels of estradiol, progesterone and AMH and decreasing the cellular apoptosis levels, while the ovarian antioxidant capacity and developing follicle numbers were significantly increased after rhLF administrations. In addition, our studies indicated that rhLF has a broad protective effect on rat ovarian functions with the potential target as GCs, which also indicates that rhLF could be applied as a promising supplement during the clinical treatment of POF.

\section{Methods}

\section{Chemicals}

Unless otherwise indicated, all chemicals, medium and supplements used in this study were purchased from Sigma Aldrich (Shanghai, China).

The freeze-dried powder of rhLF was gifted by Mr. Yunping Dai from the State Key Laboratory of Agricultural Biotechnology, China Agricultural University, Beijing, China.

The purity of rhLF was assessed using the reversedphase high-performance liquid chromatography (RPHPLC) by TSKgel protein C4-300 columns (TOSOH). For the preparation of rhLF solution, the powder of rhLF was dissolved with Dulbecco's phosphate buffered saline (DPBS) solution and the concentration of rhLF applied in this study was optimized based on the human equivalent doses calculated by previous studies $[25,36]$.

\section{Animals and POF model induction}

Eighty female Sprague Dawley (SD) rats (body weight as 200-220 g) were purchased from Beijing Vital River Laboratory Animal Technology Co., Ltd. (Beijing, China) and housed in the experimental animal room with controlled temperature $\left(20-23{ }^{\circ} \mathrm{C}\right)$ and humidity $(60 \pm 5 \%)$ under standard 12-h light/dark cycles and free access to food and water.

After acclimatization for 1 week, the body weight and vaginal smear analysis of each rat were examined prior to drug administration to ensure the normal estrous cycle (defined as $4-6 \mathrm{~d}$ in length) and comparability of all experimental data among different administration groups [63].

For the induction of POF, all rats were intraperitoneally administered with $50 \mathrm{mg} / \mathrm{kg}$ CTX (C0768, Sigma Aldrich, Shanghai, China) on the first day and then with continuous $8 \mathrm{mg} / \mathrm{kg}$ CTX for $14 \mathrm{~d}$ according to the former studies $[40,76]$.

\section{rhLF administration}

After CTX treatments, the rats were randomly assigned to four experimental groups $(n=20)$ as follows: the normal control group (NC group administrated with normal saline), low dose rhLF group (LD group, $150 \mathrm{mg} /$ $\mathrm{Kg}$ ), medium dose rhLF group (MD, $300 \mathrm{mg} / \mathrm{Kg}$ ) and high dose rhLF group (HD, $450 \mathrm{mg} / \mathrm{Kg}$ ), respectively, followed by the daily intragastric administration for 30 d.

\section{Assessment of estrous cycles}

After the intragastric administration of rhLF, the estrous cycles of 10 rats from each group was checked by vaginal smear analyses [70] with the length of an estrous cycle calculated as the average number of days between two non-consecutive days [73].

\section{Hormonal assessment}

To analysis the effect of rhLF administrations on the female reproductive functions of the CTX-induced POF rats, 10 rats at dioestrus from each group were randomly selected after estrous cycles assessment, and sacrificed by carbon dioxide with the body weight of each rat recorded and corresponding blood serum collected to analysis the hormonal levels of anti-Müllerian hormone $(\mathrm{AMH})$, estradiol and progesterone with commercial ELISA kits according to the manufacturer's instructions (E-EL-R3022 from Elabscience for AMH, PE223 from Beyotime for estradiol and PP773 from Beyotime for progesterone, Shanghai, China). To confirm the availability of experimental data, each sample was investigated in triplicate.

\section{Organ index analyses and HE staining}

To analysis the effect of rhLF administrations on the ovarian development of the CTX-induced POF rats, after blood serum collection, the ovarian wet weights of each sacrificed rat were recorded with the ovarian index (ovarian weight/body weight $\times 100 \%$ ) further analyzed, respectively.

After ovarian weight recorded, the ovaries $(n=5)$ of each sacrificed rat were individually kept in $4 \%$ paraformaldehyde solution (PFA, P1110, Solarbio, Beijing, China) for $24 \mathrm{~h}$ at room temperature for the following histological analyses.

After paraformaldehyde fixation, the ovaries were individually embedded in paraffin according to the department protocols, followed by $5 \mu \mathrm{m}$ serial sections preparation and hematoxylin and eosin staining (HE) with commercial kit (G1120, Solarbio, Beijing, China) according to the manufacturer's instructions [37].

\section{Histological analyses and follicle count}

After HE staining, the number of follicles at different stages (primary, secondary, antral and atretic follicles) in every fifth section of each sacrificed rat was recorded with random start as detailed previously $[47,48,83]$. In 
addition, the numbers of corpus lutea in every fifth section of each sacrificed rat was counted.

To ensure the accuracy of follicle stages and numbers in different groups, the follicle count was conducted by a single well-trained ovarian histologist under a blinded fashion and two other members of the group periodically evaluated the random sections.

\section{Immunohistochemistry staining}

To evaluate the effect of rhLF administrations on the proliferative abilities of follicles from the CTX-induced POF rats, Immunohistochemistry staining of Ki-67 protein was performed. Briefly, after dewaxing, gradient rehydration and antigen retrieval, the serial sections $(n=5)$ from each group were blocked with $5 \%$ bovine serum albumin (BSA, A8010, Solarbio, Beijing, China) in DPBS solution for $30 \mathrm{~min}$ at $37^{\circ} \mathrm{C}$. And the serial sections were incubated overnight at $4{ }^{\circ} \mathrm{C}$ with a rabbit anti-Ki67 antibody (with 1:300 diluted concentrations, 27,309-1-AP, Proteintech, Wuhan, China). After overnight incubation, the serial sections were rinsed with DPBS solution for three times (5 min for each time) and subsequently incubated with a goat anti-rabbit HRP secondary antibody (with 1:300 diluted concentrations, ZDR-5306, ZSGB$\mathrm{BIO}$, Beijing, China) at $25^{\circ} \mathrm{C}$ for $2 \mathrm{~h}$, respectively.

For the color reaction, the serial sections were incubated with fresh-prepared DAB substrate chromogen solution (DA1010, Solarbio, Beijing, China) at $37^{\circ} \mathrm{C}$ for 3 min and re-stained with hematoxylin solution for $5 \mathrm{~min}$. These stained sections were analyzed under a blinded fashion with a light microscope (CI-L, Nikon, Tokyo, Japan). Furthermore, the Ki-67 positive intensities in ovaries of different groups were analyzed by Image J based on the proportion of brown staining cells.

\section{Tunnel assays}

To evaluate the effect of rhLF administrations on the disruption of DNA and cellular apoptosis levels in the follicles from the CTX-induced POF rats, Tunnel assays were conducted according to the manufacturer's instructions. Briefly, after dewaxing and rehydration, $5 \mu \mathrm{m}$ serial sections of each group were stained with the Tunnel apoptosis detection (FITC) kit (40306ES20, Yeasen, Shanghai, China). The morphometric analyses of Tunnel positive cells in the follicles of each group were performed and the apoptosis levels of each group were calculated with Image J based on the proportion of Tunnel positive cells (as apoptotic cells shown with green fluorescence).

\section{The assays of ovarian ROS, SOD, MDA and TAOC levels}

To analysis the effect of rhLF administrations on the antioxidant abilities of the ovaries from CTX-induced POF rats, fresh ovaries $(n=5)$ from each group were collected for the assays of ovarian ROS, superoxide dismutase (SOD), malondialdehyde (MDA) and total antioxidative capacity (TAOC) levels. Briefly, after ovarian tissue homogenation, the levels of ROS, SOD, MDA and TAOC of different groups were detected by commercial assay kits (S0033S for ROS, S0109 for SOD, S0131 for MDA and S0121 for TAOC, Beyotime, Shanghai, China) according to the manufactures' instructions.

\section{Ovarian PCR analyses}

To analysis the effect of rhLF administrations on the ovarian gene expression levels of CTX-induced POF rats, total RNA of fresh ovaries $(n=5)$ from each group were extracted with Trizol solution (79,306, Gibco, Shanghai, China), respectively. The reversed synthesis of cDNA was carried out using a commercial Prime Script ${ }^{\mathrm{m}} \mathrm{RT}$ reagent kit (RR047A; Takara, Dalian, China), followed by Real-time PCR within a Thermo Scientific Pikoreal system by commercial kits (RR820A, Takara, Dalian, China). The qualities of PCR reactions were confirmed by the melting curves with all experiments performed in triplicate to confirm the data availability. The relative gene expression levels were calculated by the $2^{-\Delta \Delta C t}$ method with the ubiquitously expressed $\beta$-actin gene used as internal controls [37].

The primers for reverse transcription PCR and Realtime PCR analyses were shown as follows.

\section{Oct4}

Forward primer-CGAGGCCTTTCCCTCTGTTCCT; Reverse primer-TCTCTTTGTCTACCTCCCTTCCTT GC;

Foxo3

Forward primer-GGCAAAGCAGACCCTCAAACTG $A C$;

Reverse primer-TGCCCACGATGGCAGGTCAC; Bmp15

Forward primer-CCCTCCTTGCTGAAAACCCT; Reverse primer-TCAGCATGTACCTCAGGGGA; Gdf9

Forward primer-CAGGCTGGAGCCAGTGAAAA; Reverse primer-TTAGGGGTCTCACTTCGCCT; Inhibin alpha

Forward primer-ACAGGTGCCACCTGTGAGGA; Reverse primer-TGTCCCAAGGACACAGGCAC; Fshr

Forward primer-TGCAAACTTGAAGCGGCAAATC $T C$;

Reverse primer-CAAGACCCTGAGGATGTTGTACCC; Sod2

Forward primer-CTGGCCAAGGGAGATGTTAC; Reverse primer-CAGCAACTCTCCTTTGGGT; Catalase

Forward primer-GCGGATTCCTGAGAGAGTGG; 
Reverse primer-GAATCGGACGGCAATAGGAG; Gpx1

Forward primer-CAGTTCGGACATCAGGAGAAT;

Reverse primer-AGAGCGGGTGAGCCTTCT;

Bax

Forward primer-GAGGATGATTGCTGATGTGGAT

$A C$;

Reverse primer-AGTTGAAGTTGCCGTCTGC;

Bcl-2

Forward primer-GACTGAGTACCTGAACCGGCATC;

Reverse primer-CTGAGCAGCGTCTTCAGAGACA;

$\beta$-actin

Forward primer-GACTCATCGTACTCCTGCTTGCTG;

Reverse primer-GGAGATTACTGCCCTGGCTCCTA.

\section{Western blot}

To analysis the effect of rhLF administrations on the ovarian protein expression levels of CTX-induced POF rats, the protein lysates of fresh ovaries $(n=5)$ from each group were extracted with a commercial protein extraction kit (DE101, TRANS, Beijing, China), respectively, followed by the protein content measurement by $\mathrm{BCA}$ kits (163-2086, BioRad, Beijing, China) according to manufacturer's instructions.

The proteins lysates of each group were resolved on 10\% SDS-PAGE gels (P1200, Solarbio, Beijing, China) and transferred to polyvinylidene fluoride (PVDF) membranes (IPVH00010, Millipore, Beijing, China), respectively. The PVDF membranes were blocked by $10 \%$ nonfat milk in Tris- $\mathrm{HCl}$ solution containing $0.1 \%$ Tween-20 (TBST) and separately incubated with the following primary antibodies at $4{ }^{\circ} \mathrm{C}$ overnight: a rabbit anti-Bax antibody (with 1:1000 diluted concentration, 50,599-2-lg, Proteintech, Wuhan, China), a rabbit anti-Bcl-2 antibody (with 1:1000 diluted concentration, 12,789-1-AP, Proteintech, Wuhan, China), a rabbit anti-Caspase 3 antibody (with 1:1000 diluted concentration 19,677-1-AP, Proteintech, Wuhan, China) and a rabbit anti-GAPDH antibody (with 1:1000 diluted concentration, ab8245, Abcam, Shanghai, China) as loading controls, respectively.

The membranes were washed three times with TBST solution and incubated with goat anti-rabbit HRP secondary antibodies (with 1:1000 diluted concentration, ZDR5306, ZSGB-BIO, Beijing, China) at room temperature for $1 \mathrm{~h}$, respectively. The blots were visualized with enhanced chemiluminescence solutions (ECL, W1001, Promega, Beijing, China) and recorded within a ChampChemi 610 Plus system. Furthermore, the protein expression levels normalized to GAPDH were analyzed by Image J.

\section{Isolation and culture of primary rat granulosa cells}

The isolation and culture of primary rat granulosa cells (GCs) were conducted as previously reported [6, 61]. Briefly, the immature female rats ( $21 \mathrm{~d}$ old) were injected intraperitoneally with $10 \mathrm{IU} / \mathrm{mL}$ pregnant mare serum gonadotropin (PMSG, Sansheng, Ningbo, China) and sacrificed by cervical dislocation after $48 \mathrm{~h}$. The bilateral ovaries were immediately collected and washed in DPBS solution supplemented with $100 \mathrm{U} / \mathrm{mL}$ penicillin/ streptomycin antibiotics (P/S, 15070063, Invitrogen, China). After the removal of surrounding fat tissues, the ovaries were placed in the ice-cold DPBS solution and punctured with 27-gauge needles. The ovaries were then digested with hyaluronidase solution (H3605, Sigma Aldrich, Shanghai, China), followed by the collection of primary rat GCs. after cell counting, the primary rat GCs were cultured in 24 well plates (Corning, Beijing, China) at a density of $5 \times 10^{4}$ cells/ well in a humidified atmosphere of $5 \% \mathrm{CO}_{2}$ and $95 \%$ air at $37^{\circ} \mathrm{C}$. For the following studies, the 24 well plates were pre-plated with sterile cover-slips. The culture medium for primary rat GCs was the DMEM/F12 medium (11,320,033, Gibco, Shanghai, China) supplemented with $15 \%$ FBS $(10,091$, 148, Gibco, Shanghai, China) and $100 \mathrm{U} / \mathrm{mL} \mathrm{P} / \mathrm{S}$.

\section{Rat GCs treatments and experimental group settings}

The activity of CTX requires a 4-hydroxylation reaction in the liver tissues, leading to the incapable applications of CTX in vitro. 4-hydroperoxy cyclophosphamide (4$\mathrm{HC}$ ), as the active in vitro metabolite of CTX, was applied for the following treatments of rat GCs $[59,80]$.

During the primary rat GCs culture in vitro, $100 \mu \mathrm{g} /$ $\mathrm{mL}$ 4-HC solution (gifted by Mrs. Xiulan Su from the Key Laboratory of Medical Cell Biology, Clinical Medicine Research Center, the Affiliated Hospital of Inner Mongolia Medical University) was supplemented to the culture medium of primary rat GCs based on the former studies about the rat GCs toxicity of 4-HC [80]. During the $4-\mathrm{HC}$ treatments for $24 \mathrm{~h}$, the culture medium of primary rat GCs was also supplemented with DPBS (the negative control group, rhLF 0 group), $100 \mu \mathrm{g} / \mathrm{mL}$ rhLF (rhLF 100 group), $200 \mu \mathrm{g} / \mathrm{mL}$ rhLF (rhLF 200 group) and $300 \mu \mathrm{g} / \mathrm{mL}$ rhLF (rhLF 300 group), respectively, followed by the cell culture in a $\mathrm{CO}_{2}$ incubator $\left(37.0^{\circ} \mathrm{C}, 5 \% \mathrm{CO}_{2}\right)$.

\section{ROS assay of rat GCs}

To analysis the ameliorative effect of rhLF treatments on the antioxidant abnormalities of rat GCs caused by 4$\mathrm{HC}$ in vitro, the ROS production levels of primary rat GCs after drug treatments were detected with ROS assay kits (S0033, Beyotime, Shanghai, China) according to the manufacturer's instructions.

Briefly, the primary rat GCs pre-plated on sterile cover-slips were washed three times with DPBS solution and incubated with $10 \mu \mathrm{M}$ dichlorofluorescein diacetate (DCFH-DA, S0033, Beyotime, Shanghai, China) for 30 min at $37^{\circ} \mathrm{C}$, respectively. After DCFH-DA incubation, the cells were individually re-stained with $10 \mu \mathrm{g} / \mathrm{mL}$ 
Hoechst 33432 solutions (C0030, Solarbio, Beijing, China) for $5 \mathrm{~min}$ at $37^{\circ} \mathrm{C}$. The cellular fluorescence staining densities in different groups were analyzed by an inverted fluorescence microscopy (Ti, Nikon, Tokyo, Japan).

\section{EdU staining of rat GCs}

To analysis the ameliorative effect of rhLF treatments on the abnormal proliferation of rat GCs caused by 4-HC in vitro, the proliferation of rat GCs after drug treatments were detected with EDU (5-ethynyl-2'-deoxyuridine) assay kits (C0071S, Beyotime, Shanghai, China) according to the manufacturer's instructions.

Briefly, the primary rat GCs pre-plated on sterile cover-slips were washed three times with DPBS solution and incubated with EdU solution for $2 \mathrm{~h}$, followed by the re-staining with $10 \mu \mathrm{g} / \mathrm{mL}$ Hoechst 33432 solutions for 5 min at $37^{\circ} \mathrm{C}$. After cover-slip sealing, the cellular fluorescence staining densities in different groups were analyzed by an inverted fluorescence microscopy.

\section{PCR analyses of rat GCs}

To analysis the ameliorative effect of rhLF treatments on the abnormal gene expression levels of rat GCs caused by 4-HC in vitro, the expression levels of Inhibin alpha, Fshr, Sod2, Catalase, Gpx1, Bax and Bcl-2 in rat GCs after drug treatments were detected with PCR analyses according to the methodologies in the section of ovarian PCR analyses.

\section{Fertility assessment after rhLF administrations}

The fertility assessment was performed to assess the potential effects of rhLF on the fertilities of CTX-induced POF rats. In order to avoid the risk of CTX-related fetal malformations [43], 4 weeks after rhLF administrations, 10 rat of each group were individually paired with normal male rats (12 weeks old) of proven fertility at a 1:1 ratio, followed by the mating examination (appearance of the vaginal plug). On the day when copulation was confirmed, the successful mating days were recorded with the female rat of each group housed individually. On postnatal day (PND) 0, the gestation length of each group and the offspring data (number of pups per litter and pup weight) were recorded.

Every other week, three female offspring of each group were humanely sacrificed by carbon dioxide with the body weight recorded and hormonal levels of estradiol and progesterone analyzed. Furthermore, the ovaries of each sacrificed offspring were collected, followed by the analyses of ovarian index, preparation of paraffin sections and HE staining.

\section{Statistical analyses}

In this study, the results were expressed as means \pm SEM. One-way analyses of variance (ANOVA) with IBM SPSS statistic's software 19.0 were applied to analysis the experimental data among different groups, followed by the LSD comparison post-test. $P<0.05$ was considered as significant.

\section{Abbreviations \\ POF: Premature ovarian failure; AMH: Anti-Müllerian hormone; MDR: Multidrug resistance; ROS: Reactive oxygen species; \\ CTX: Cyclophosphamide; RP-HPLC: Reversed-phase high-performance liquid chromatography; DPBS: Dulbecco's phosphate buffered saline; HE: Hematoxylin and eosin staining; SOD: Superoxide dismutase; MDA: Malondialdehyde; TAOC: Total antioxidative capacity; \\ PVDF: Polyvinylidene fluoride; TBST: Tris- $\mathrm{HCl}$ solution containing $0.1 \%$ Tween- 20; PND: Postnatal day; ANOVA: One-way analyses of variance; IVF: In vitro fertilization; MPTP: 1-methyl-4-phenyl-1,2,3,6-tetrahydropyridine; DMT1: Divalent metal transporter; TFR: Transferrin receptor; FSH: Follicle stimulating hormone; LH: Luteinizing hormone; ESCs: Embryonic stem cells; iPSCs: Induced pluripotent stem cells; GCs: Granulosa cells; PMSG: Pregnant mare serum gonadotropin; P/S: Penicillin/streptomycin antibiotics; 4-HC: 4- hydroperoxy cyclophosphamide; DCFH-DA: Dichlorofluorescein diacetate; EDU: 5-ethynyl-2'-deoxyuridine}

\section{Acknowledgements}

We would like to thank Mr. Shu Zhang from the department of Pathology, Affiliated Hospital of Nantong University and Mr. Hao Yu from the clinical laboratory of Chifeng hospital for their technical support.

\section{Authors' contributions}

Data curation, Shubin Li, Mengnan Liu and Gang Liu; Formal analysis, Hongmeng Ma, Gang Liu and Yanfeng Dai; Funding acquisition, Mengnan Liu, Gang Liu and Yanfeng Dai; Investigation, Shubin Li, Mengnan Liu, Hongmeng Ma, Qin Jin, Yuzhen Ma, Chunyu Wang, Jingyu Ren and Gang Liu; Methodology, Shubin Li, Mengnan Liu, Qin Jin, Yuzhen Ma, Chunyu Wang, Jingyu Ren and Gang Liu; Project administration, Yanfeng Dai; Resources, Yanfeng Dai; Supervision, Gang Liu and Yanfeng Dai; Writingoriginal draft, Hongmeng Ma and Gang Liu; Writing-review \& editing, Yanfeng Dai. The author (s) read and approved the final manuscript.

\section{Funding}

This work was supported by the National Natural Science Foundation of China (82060567 to Gang Liu), the Natural Science Foundation of Inner Mongolia (2020BS08014 to Gang Liu and 2017ZD04 to Yanfeng Dai) and Chifeng Technology Research Project (SZR2019047 to Mengnan Liu).

\section{Availability of data and materials}

We declared that materials described in the manuscript, including all relevant raw data, will be freely available to any scientist wishing to use them for non-commercial purposes, without breaching participant confidentiality.

Ethics approval and consent to participate

The permission of this study was granted by the Animal Care Committee of Inner Mongolia University (IACUC Issue No.20201205 to Gang Liu).

Consent for publication

Not applicable.

\section{Competing interests}

The authors report no conflicts of interest in this work.

\section{Author details}

${ }^{1}$ College of Life Science, Inner Mongolia University, 235 West University Road, Hohhot 010021, Inner Mongolia, China. ${ }^{2}$ Centre of Reproductive Medicine, Inner Mongolia Hospital, 20 Zhaowuda Road, Hohhot 010021, Inner Mongolia, China. ${ }^{3}$ Clinical Medicine Research Center, Chifeng municipal hospital, 1 Zhaowuda Road, Chifeng 024000, Inner Mongolia, China. 
${ }^{4}$ Department of Pathology, Affiliated Hospital of Nantong University, 20 Xisi Road, Nantong 226001, Jiangsu, China. ${ }^{5}$ Key Laboratory of Medical Cell Biology, Clinical Medicine Research Center, the Affiliated Hospital of Inner Mongolia Medical University, 1 Tongdao North Street, Hohhot 010050, Inner Mongolia, China.

\section{Received: 12 October 2020 Accepted: 27 December 2020}

Published online: 21 January 2021

\section{References}

1. Ashton TM, McKenna WG, Kunz-Schughart LA, Higgins GS. Oxidative phosphorylation as an emerging target in Cancer therapy. Clin Cancer Res. 2018:24(11):2482-90.

2. Beck-Peccoz P, Persani L. Premature ovarian failure. Orphanet J Rare Dis. 2006;1:9.

3. Bray F, Ferlay J, Soerjomataram I, Siegel RL, Torre LA, Jemal A. Global cancer statistics 2018: GLOBOCAN estimates of incidence and mortality worldwide for 36 cancers in 185 countries. CA Cancer J Clin. 2018;68(6):394-424.

4. Chapman C, Cree L, Shelling AN. The genetics of premature ovarian failure: current perspectives. Int J Women's Health. 2015;7:799-810.

5. Chatterjee R, Helal M, Mobberley M, Ryder T, Bajoria R. Impaired steroidogenesis and apoptosis of granulosa-luteal cells in primary culture induced by cis-platinum. Am J Obstetr Gynecol. 2014;210(3):252.e1-7.

6. Chowdhury I, Xu W, Stiles JK, Zeleznik A, Yao X, Matthews R, et al. Apoptosis of rat granulosa cells after staurosporine and serum withdrawal is suppressed by adenovirus-directed overexpression of prohibitin. Endocrinology. 2007;148(1):206-17.

7. Conesa C, Calvo M, Sánchez L. Recombinant human lactoferrin: a valuable protein for pharmaceutical products and functional foods. Biotechnol Adv. 2010;28(6):831-8

8. Cubillos-Ruiz JR, Bettigole SE, Glimcher LH. Tumorigenic and immunosuppressive effects of endoplasmic reticulum stress in Cancer. Cell. 2017;168(4):692-706

9. Cui Q, Wang JQ, Assaraf $Y G$, Ren L, Gupta $P$, Wei $L$, et al. Modulating ROS to overcome multidrug resistance in cancer. Drug Resist Updat. 2018:41:1-25.

10. Dagogo-Jack I, Shaw AT. Tumour heterogeneity and resistance to cancer therapies. Nat Rev Clin Oncol. 2018;15(2):81-94.

11. Del Mastro L, Ceppi M, Poggio F, Bighin C, Peccatori F, Demeestere I, et al. Gonadotropin-releasing hormone analogues for the prevention of chemotherapy-induced premature ovarian failure in cancer women: systematic review and meta-analysis of randomized trials. Cancer Treat Rev. 2014;40(5):675-83.

12. Duffy DM, Ko C, Jo M, Brannstrom M, Curry TE. Ovulation: parallels with inflammatory processes. Endocr Rev. 2019;40(2):369-416.

13. Dumesic DA, Hoyos LR, Chazenbalk GD, Naik R, Padmanabhan V, Abbott $\mathrm{DH}$. Mechanisms of intergenerational transmission of polycystic ovary syndrome. Reproduction. 2020;159(1):R1-R13.

14. Dynes J, Osz K, Hooper A, Petrik J. Low-dose metronomic delivery of cyclophosphamide is less detrimental to granulosa cell viability, ovarian function, and fertility than maximum tolerated dose delivery in the mouse. Biol Reprod. 2017;97(3):449-65.

15. El-Ashker M, Risha E, Abdelhamid F, Ateya A. Potential immune modulating properties and antioxidant activity of supplementing commercially available lactoferrin and/or Lactobacillus sp. in healthy Ossimi lambs. Pol J Vet Sci. 2018;21(4):705-13.

16. Emadi A, Jones RJ, Brodsky RA. Cyclophosphamide and cancer: golden anniversary. Nat Rev Clin Oncol. 2009;6(11):638-47.

17. Farid AS, El Shemy MA, Nafie E, Hegazy AM, Abdelhiee EY. Antiinflammatory, anti-oxidant and hepatoprotective effects of lactoferrin in rats. Drug Chem Toxicol. 2019;(2):1-8.

18. Fuchs-Tarlovsky V. Role of antioxidants in cancer therapy. Nutrition. 2013; 29(1):15-21.

19. Ghobadi E, Moloudizargari M, Asghari MH, Abdollahi M. The mechanisms of cyclophosphamide-induced testicular toxicity and the protective agents. Expert Opin Drug Metab Toxicol. 2017;13(5):525-36.

20. Haider T, Tiwari R, Vyas SP, Soni V. Molecular determinants as therapeutic targets in cancer chemotherapy: an update. Pharmacol Ther. 2019;200:85109.

21. Hassoun LA, Sivamani RK. A systematic review of lactoferrin use in dermatology. Crit Rev Food Sci Nutr. 2017;57(17):3632-9.
22. Hayashi K, Ogushi S, Kurimoto K, Shimamoto S, Ohta H, Saitou M, et al. Science (New York, NY). 2012;338(6109):971-5.

23. Hikabe O, Hamazaki N, Nagamatsu G, Obata Y, Hirao Y, Hamada N, et al. Reconstitution in vitro of the entire cycle of the mouse female germ line. Nature. 2016;539(7628):299-303.

24. Horiuchi Y, Higuchi T, Tatsumi K, Takakura K, Fujii S, Konishi I. Lactoferrin is associated with a decrease in oocyte depletion in mice receiving cyclophosphamide. Fertil Steril. 2009;91(5 Suppl):2069-2078.e1.

25. Hu W, Zhao J, Wang J, Yu T, Wang J, Li N. Transgenic milk containing recombinant human lactoferrin modulates the intestinal flora in piglets. Biochem Cell Biol. 2012;90(3):485-96.

26. Huang $T$, Song $C$, Zheng $L$, Xia L, Li Y, Zhou Y. The roles of extracellular vesicles in gastric cancer development, microenvironment, anti-cancer drug resistance, and therapy. Mol Cancer. 2019;18(1):62.

27. Iglesias-Fiqueroa BF, Siqueiros-Cendón TS, Gutierrez DA, Aguilera RJ, Espinoza-Sánchez EA, Arévalo-Gallegos S, et al. Recombinant human lactoferrin induces apoptosis, disruption of F-actin structure and cell cycle arrest with selective cytotoxicity on human triple negative breast cancer cells. Apoptosis. 2019;24(7-8):562-77.

28. Jans DA, Martin AJ, Wagstaff KM. Inhibitors of nuclear transport. Curr Opin Cell Biol. 2019;58:50-60.

29. Jeelani R, Khan SN, Shaeib F, Kohan-Ghadr HR, Aldhaheri SR, Najafi T, et al Cyclophosphamide and acrolein induced oxidative stress leading to deterioration of metaphase II mouse oocyte quality. Free Radic Biol Med. 2017;110:11-8

30. Kokcu A. Premature ovarian failure from current perspective. Gynecol Endocrinol. 2010;26(8):555-62.

31. Kopecka J, Trouillas P, Gašparović A, Gazzano E, Assaraf YG, Riganti C. Phospholipids and cholesterol: inducers of cancer multidrug resistance and therapeutic targets. Drug ResistUpdat. 2020:49:100670.

32. Kunzelmann K, Ousingsawat J, Benedetto R, Cabrita I, Schreiber R. Contribution of Anoctamins to Cell Survival and Cell Death. Cancers. 2019; 11(3):382

33. La Vecchia C. Hormone replacement therapy, breast and endometrial cancer. Eur J Cancer Prev. 1996;5(5):414-6.

34. Legrand D, Pierce A, Elass E, Carpentier M, Mariller C, Mazurier J. Lactoferrin structure and functions. Adv Exp Med Biol. 2008;606:163-94.

35. Lheureux S, Braunstein M, Oza AM. Epithelial ovarian cancer: evolution of management in the era of precision medicine. CA Cancer J Clin. 2019;69(4): 280-304.

36. Li Q, Hu W, Zhao J, Wang J, Dai Y, Zhao Y, et al. Supplementation transgenic cow's milk containing recombinant human lactoferrin enhances systematic and intestinal immune responses in piglets. Mol Biol Rep. 2014; 41(4):2119-28.

37. Liu G, Li S, Yuan H, Hao M, Wurihan, Yun Z, et al. Effect of sodium alginate on mouse ovary vitrification. Theriogenology. 2018;113:78-84.

38. Liu H, Wu H, Zhu N, Xu Z, Wang Y, Qu Y, et al. Lactoferrin protects against iron dysregulation, oxidative stress, and apoptosis in 1-methyl-4-phenyl1,2,3,6-tetrahydropyridine (MPTP)-induced Parkinson's disease in mice. J Neurochem. 2020;152(3):397-415

39. Liu T, Li Q, Wang S, Chen C, Zheng J. Transplantation of ovarian granulosalike cells derived from human induced pluripotent stem cells for the treatment of murine premature ovarian failure. Mol Med Rep. 2016;13(6): $5053-8$

40. Liu T, Wang S, Li Q, Huang Y, Chen C, Zheng J. Telocytes as potential targets in a cyclophosphamide-induced animal model of premature ovarian failure. Mol Med Rep. 2016;14(3):2415-22.

41. Maacha S, Bhat AA, Jimenez L, Raza A, Haris M, Uddin S, et al. Extracellular vesicles-mediated intercellular communication: roles in the tumor microenvironment and anti-cancer drug resistance. Mol Cancer. 2019;18(1):55.

42. Martorell P, Llopis S, Gonzalez N, Ramón D, Serrano G, Torrens A, et al. A nutritional supplement containing lactoferrin stimulates the immune system, extends lifespan, and reduces amyloid $\beta$ peptide toxicity in Caenorhabditis elegans. Food Sci Nutr. 2017:5(2):255-65.

43. Meirow D, Epstein M, Lewis H, Nugent D, Gosden RG. Administration of cyclophosphamide at different stages of follicular maturation in mice: effects on reproductive performance and fetal malformations. Hum Reprod. 2001:16(4):632-7.

44. Mulder AM, Connellan PA, Oliver CJ, Morris CA, Stevenson LM. Bovine lactoferrin supplementation supports immune and antioxidant status in healthy human males. Nutr Res. 2008;28(9):583-9. 
45. Nayak G, Rao A, Mullick P, Mutalik S, Kalthur SG, Adiga SK, et al. Ethanolic extract of Moringa oleifera leaves alleviate cyclophosphamide-induced testicular toxicity by improving endocrine function and modulating cell specific gene expression in mouse testis. J Ethnopharmacol. 2020;259:112922.

46. Nie X, Dai Y, Zheng Y, Bao D, Chen Q, Yin Y, et al. Establishment of a mouse model of premature ovarian failure using consecutive superovulation. Cell Physiol Biochem. 2018;51(5):2341-58.

47. Oner G, Ozcelik B, Ozgun MT, Ozturk F. The effects of metformin and letrozole on endometrium and ovary in a rat model. Gynecol Endocrinol. 2011;27(12):1084-6.

48. Ozcelik B, Turkyilmaz C, Ozgun MT, Serin IS, Batukan C, Ozdamar S, et al. Prevention of paclitaxel and cisplatin induced ovarian damage in rats by a gonadotropin-releasing hormone agonist. Fertil Steril. 2010;93(5):1609-14.

49. Park SY, Jeong AJ, Kim GY, Jo A, Lee JE, Leem SH, et al. Lactoferrin protects human Mesenchymal stem cells from oxidative stress-induced senescence and apoptosis. J Microbiol Biotechnol. 2017;27(10):1877-84.

50. Pastori V, Tavazzi S, Lecchi M. Lactoferrin-loaded contact lenses: eye protection against oxidative stress. Cornea. 2015;34(6):693-7.

51. Pavin NF, Izaguirry AP, Soares MB, Spiazzi CC, Mendez ASL, Leivas FG, et al Tribulus terrestris protects against male reproductive damage induced by cyclophosphamide in mice. Oxidative Med Cell Longev. 2018;2018:5758191.

52. Pérez-Herrero E, Fernández-Medarde A. Advanced targeted therapies in cancer: drug nanocarriers, the future of chemotherapy. Eur J Pharm Biopharm. 2015;93:52-79.

53. Perez MF, Lehner B. Intergenerational and transgenerational epigenetic inheritance in animals. Nat Cell Biol. 2019;21(2):143-51.

54. Robey RW, Pluchino KM, Hall MD, Fojo AT, Bates SE, Gottesman MM Revisiting the role of $\mathrm{ABC}$ transporters in multidrug-resistant cancer. Nat Rev Cancer. 2018;18(7):452-64.

55. Roti Roti EC, Salih SM. Dexrazoxane ameliorates doxorubicin-induced injury in mouse ovarian cells. Biol Reprod. 2012;86(3):96.

56. Smart E, Lopes F, Rice S, Nagy B, Anderson RA, Mitchell RT, et al. Chemotherapy drugs cyclophosphamide, cisplatin and doxorubicin induce germ cell loss in an in vitro model of the prepubertal testis. Sci Rep. 2018;8(1):1773.

57. Spears N, Lopes F, Stefansdottir A, Rossi V, De Felici M, Anderson RA, et al. Ovarian damage from chemotherapy and current approaches to its protection. Hum Reprod Update. 2019;25(6):673-93.

58. Suzuki YA, Lönnerdal B. Baculovirus expression of mouse lactoferrin receptor and tissue distribution in the mouse. Biometals. 2004;17(3):301-9.

59. Teicher BA, Holden SA, Goff DA, Wright JE, Tretyakov O, Ayash LJ. Antitumor efficacy and pharmacokinetic analysis of 4-hydroperoxycyclophosphamide in comparison with cyclophosphamide +/- hepatic enzyme effectors. Cancer Chemother Pharmacol. 1996:38(6):553-60.

60. Telang S. Lactoferrin: A Critical Player in Neonatal Host Defense. Nutrients. 2018;10(9):1228.

61. Thompson WE, Sanbuissho A, Lee GY, Anderson E. Steroidogenic acute regulatory (StAR) protein (p25) and prohibitin (p28) from cultured rat ovarian granulosa cells. J Reprod Fertil. 1997;109(2):337-48.

62. Tripathi DN, Jena GB. Astaxanthin inhibits cytotoxic and genotoxic effects of cyclophosphamide in mice germ cells. Toxicology. 2008;248(2-3):96-103.

63. Tropp J, Markus EJ. Effects of mild food deprivation on the estrous cycle of rats. Physiol Behav. 2001;73(4):553-9.

64. Turner JG, Dawson J, Sullivan DM. Nuclear export of proteins and drug resistance in cancer. Biochem Pharmacol. 2012:83(8):1021-32.

65. Uyar A, Torrealday S, Seli E. Cumulus and granulosa cell markers of oocyte and embryo quality. Fertil Steril. 2013;99(4):979-97.

66. Wakabayashi H, Yamauchi K, Takase M. Lactoferrin research, technology and applications. Int Dairy J. 2006;16:1241-51.

67. Wang B, Timilsena YP, Blanch E, Adhikari B. Lactoferrin: structure, function, denaturation and digestion. Crit Rev Food Sci Nutr. 2019;59(4):580-96.

68. Wang WP, ligo M, Sato J, Sekine K, Adachi I, Tsuda H. Activation of intestinal mucosal immunity in tumor-bearing mice by lactoferrin. Jpn J Cancer Res. 2000; 91(10):1022-7.

69. Weinberg SE, Chandel NS. Targeting mitochondria metabolism for cancer therapy. Nat Chem Biol. 2015;11(1):9-15.

70. Westwood FR. The female rat reproductive cycle: a practical histological guide to staging. Toxicol Pathol. 2008;36(3):375-84

71. Wu T, Dai Y. Tumor microenvironment and therapeutic response. Cancer Lett. 2017;387:61-8.

72. Xu S-F, Zhang Y-H, Wang S, Pang Z-Q, Fan Y-G, Li J-Y, et al. Lactoferrin ameliorates dopaminergic neurodegeneration and motor deficits in MPTPtreated mice. Redox Biol. 2019;21:101090.
73. Xu X, Tan Y, Jiang G, Chen X, Lai R, Zhang L, et al. Effects of Bushen Tianjing recipe in a rat model of tripterygium glycoside-induced premature ovarian failure. Chin Med. 2017;12:10

74. Yalçin AS. Emerging therapeutic potential of whey proteins and peptides. Curr Pharm Des. 2006;12(13):1637-43.

75. Yanaihara A, Mitsukawa K, Iwasaki S, Otsuki K, Kawamura T, Okai T. High concentrations of lactoferrin in the follicular fluid correlate with embryo quality during in vitro fertilization cycles. Fertil Steril. 2007;87(2):279-82.

76. Yang M, Lin L, Sha C, Li T, Zhao D, Wei H, et al. Bone marrow mesenchymal stem cell-derived exosomal miR-144-5p improves rat ovarian function after chemotherapy-induced ovarian failure by targeting PTEN. Lab Investig. 2020; 100(3):342-52.

77. Yang Y, Karakhanova S, Hartwig W, D'Haese JG, Philippov PP, Werner J, et al. Mitochondria and mitochondrial ROS in Cancer: novel targets for anticancer therapy. J Cell Physiol. 2016;231(12):2570-81.

78. Yasueda A, Urushima H, Ito T. Efficacy and interaction of antioxidant supplements as adjuvant therapy in Cancer treatment: a systematic review. Integr Cancer Ther. 2016;15(1):17-39.

79. Yorgun H, Tokgözoğlu L, Canpolat U, Gürses KM, Bozdağ G, Yapıcı Z, et al. The cardiovascular effects of premature ovarian failure. Int J Cardiol. 2013; 168(1):506-10.

80. Yuksel A, Bildik G, Senbabaoglu F, Akin N, Arvas M, Unal F, et al. The magnitude of gonadotoxicity of chemotherapy drugs on ovarian follicles and granulosa cells varies depending upon the category of the drugs and the type of granulosa cells. Hum Reprod. 2015;30(12):2926-35.

81. Zhang Q, Sun J, Huang Y, Bu S, Guo Y, Gu T, et al. Human amniotic epithelial cell-derived Exosomes restore ovarian function by transferring MicroRNAs against apoptosis. Mol Ther Nucleic Acids. 2019;16:407-18.

82. Zhang T, He WH, Feng LL, Huang HG. Effect of doxorubicin-induced ovarian toxicity on mouse ovarian granulosa cells. Regul Toxicol Pharmacol. 2017;86: $1-10$.

83. Zhang X, Ji M, Tan X, Yu K, Xu L, Chen G, et al. Role of epigenetic regulation of Igf2 and H19 in 2,3,7,8-Tetrachlorobenzo-p-dioxin (TCDD)-induced ovarian toxicity in offspring rats. Toxicol Lett. 2019;311:98-104.

84. Ziech D, Franco R, Georgakilas AG, Georgakila S, Malamou-Mitsi V, Schoneveld $\mathrm{O}$, et al. The role of reactive oxygen species and oxidative stress in environmental carcinogenesis and biomarker development. Chem Biol Interact. 2010;188(2):334-9.

\section{Publisher's Note}

Springer Nature remains neutral with regard to jurisdictional claims in published maps and institutional affiliations.

\section{Ready to submit your research? Choose BMC and benefit from:}

- fast, convenient online submission

- thorough peer review by experienced researchers in your field

- rapid publication on acceptance

- support for research data, including large and complex data types

- gold Open Access which fosters wider collaboration and increased citations

- maximum visibility for your research: over $100 \mathrm{M}$ website views per year

At BMC, research is always in progress.

Learn more biomedcentral.com/submissions 